\title{
Research Paper: \\ Responsiveness of Impairment-based Outcome Measures in Individuals With Anterior Cruciate Ligament Reconstruction Following Physiotherapy
}

Neda Mostafaee $^{1}$, Hossein Negahban ${ }^{1,2}$, Mohammad Jafar Shaterzadeh Yazdi ${ }^{3}$, Shahin Goharpey ${ }^{3}$, Mohammad Mehravar ${ }^{3}$, Nahid Pirayeh ${ }^{3^{*}} \mathrm{C}$

1. Department of Physical Therapy, School of Paramedical Sciences, Mashhad University of Medical Sciences, Mashhad, Iran

2. Orthopedic Research Center, Mashhad University of Medical Sciences, Mashhad, Iran

3. Musculoskeletal Rehabilitation Research Center, Ahvaz Jundishapur University of Medical Sciences, Ahvaz, Iran.

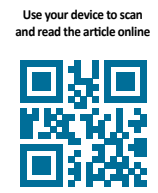

dtation Mostafaee N, Negahban H, Shaterzadeh Yazdi MJ, Goharpey Sh, Mehravar M, Pirayeh N. [Responsiveness of Impairment-based Outcome Measures in Individuals With Anterior Cruciate Ligament Reconstruction Following Physiotherapy (Persian)]. Archives of Rehabilitation. 2021; 22(2):228-245. https://doi.org/10.32598/RJ.22.2.3280.1

https://doi.org/10.32598/RJ.22.2.3280.1
Received: 08 Dec 2020

Accepted: 27 Jan 2021

Available Online: $01 \mathrm{Jul} 2021$
Keywords:

Responsiveness, Anterior cruciate Ligament reconstruction, Range of motion, Muscle strength, Balance

\begin{abstract}
Objective The primary purpose of physiotherapy in patients with Anterior Cruciate Ligament (ACL) reconstruction is to reduce pain, improve Range of Motion (ROM), muscle strength, and balance after surgery. Thus, therapists need outcome measurement tools with acceptable validity, reproducibility, and responsiveness to assess these changes after the intervention. This study investigates the responsiveness of the tools used to measure pain, ROM, knee muscle strength, and dynamic balance. We also want to determine minimal clinically significant change for these outcomes in patients with $A C L$ reconstruction after physiotherapy.

Materials \& Methods The study participants were 54 young patients with ACL reconstruction evaluated using impairment-based outcome measures of visual analog scale, goniometer, hand-held dynamometer, and Star Excursion Balance Test (SEBT) at baseline ( 2 weeks after ACL reconstruction ) and 4 weeks after physiotherapy. At the second phase of evaluation, the participants also completed the 7-point global change rating scale. For assessing the responsiveness of the tools, we used the Receiver Operating Characteristics (ROC) curve and correlation analysis

Results Analyzing the ROC curve showed that the knee extension ROM, quadriceps and hamstring strength, and medial and posteromedial directions under SEBT had acceptable responsiveness $(>0.70)$. The Spearman correlation coefficient between the scores obtained for these outcomes and the score of 7 -point global change rating scale were significant in the range of $0.36-0.51$. For all the study outcome measurement tools, minimal significant clinical changes were reported.

Conclusion The results of this study support the responsiveness of goniometer for knee extension ROM, hand-held dynamometer for quadriceps and hamstring strength, and SEBT for dynamic balance at medial and posteromedial directions in the assessment of clinical changes in patients with $A C L$ reconstruction. The reported minimal significant clinical changes for each tool can help the clinicians and researchers to decide on determining the actual significant change in the ACL patient's clinical conditions after physiotherapy.
\end{abstract}

* Corresponding Author:

Nahid Pirayeh, PhD.

Address: Musculoskeletal Rehabilitation Research Center, Ahvaz Jundishapur University of Medical Sciences, Ahvaz, Iran.

Tel: +98 (916) 3054015

E-Mail: nahid_pt8287@yahoo.com 


\section{Extended Abstract}

\section{Introduction}

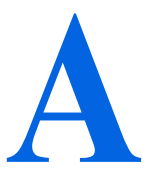

nterior Cruciate Ligament (ACL) rupture is one of the most common knee joint injuries, especially in active and young people. It accounts for about $50 \%$ of knee injuries. This ligament is usually damaged during recreational or competitive sports activities [1]. ACL reconstruction surgery is usually recommended following an ACL rupture to restore the knee mechanical stability and achieve normal knee function $[1,2]$. Studies have shown that many people still do not resume normal knee function after ACL reconstruction and report disorders such as knee instability, pain, decreased Range of Motion (ROM), defects in quadriceps and hamstrings, and neuromuscular dysfunction, which can lead to very different consequences among these people $[3,4]$. Therefore, rehabilitation interventions are essential for patients with ACL reconstruction to reduce pain, improve ROM, muscle strength, dynamic and functional stability of the reconstructed knee, balance, and finally help return the patient to functional activities [5]. According to systematic review studies, the most common disorder-based outcome measures used to evaluate the effect of treatment and determine the success of treatment in randomized clinical trials on patients with ACL reconstruction are knee pain, ROM, hamstring, and quadriceps muscle strength, and dynamic balance using Star Excursion Balance Test (SEBT) [6-8].

In this regard, therapists need repetitive, valid, and responsive tests (sensitive to change) to detect significant changes during the treatment process $[9,10]$. Responsiveness is an essential feature of a measurement tool and can detect the least clinically significant change in a patient's health status over time. Responsiveness includes internal and external types $[11,12]$. Internal responsiveness refers to the ability of a tool to detect changes over time, regardless of whether these changes are clinically significant or not $[13,14]$. External responsiveness indicates how much of a change in the instrument has occurred in a given period in accordance with the change in the reference instrument. The reference measurement is based on the retrospective 7-point Likert scale [15]. Although clinical trial studies have repeatedly used pain, ROM, quadriceps, and hamstring muscle strength, and dynamic balance control to assess patients' recovery from ACL reconstruction and the effect of treatment, no study has yet examined the responsiveness of these measures. Therefore, this study aims to investigate the responsiveness of tools measuring pain, ROM, quadriceps and hamstring muscle strength, and dynamic balance control in patients with ACL reconstruction following a rehabilitation treatment.

\section{Materials and Methods}

This research is a prospective cohort study. The study participants were 54 patients with an ACL injury who had undergone reconstructive surgery. The inclusion criteria were being 18-45 years old and passing 2 weeks from the ACL reconstruction surgery. The exclusion criteria were having a history of diabetes, neurological disorder, inflammatory arthritis, malignancy, musculoskeletal pain, musculoskeletal injury other than ACL rupture, and bilateral ACL rupture.

\section{Study measures}

The Visual Analog Scale (VAS) was used to assess pain outcomes. It consists of a $10-\mathrm{cm}$ horizontal line, with the left-hand side labeled "no pain" and the right-hand side labeled "most intense pain imaginable". The VAS score was determined by measuring in centimeters from the left-hand side to the point that the patient marks [16].

A goniometer was used to measure the passive ROM of the knee in the supine position during flexion and extension. Thus, the amplitude of knee flexion and extension was recorded [16].

To evaluate the isometric strength of quadriceps and hamstrings, a manual tension/compression dynamometer (Danesh Salar Iranian Company, Iran) was used. The dynamometer was placed just above the malleolus in the anterior and posterior parts of the leg, and the person was asked to press the involved leg against the strap as much as possible. The primary test was performed by taking maximum contraction of quadriceps and hamstring muscles with 3 repetitions during maximum effort from each person. There was a 30 -second rest interval. The strength values obtained from the three maximum contractions of each muscle group were averaged [17].

To evaluate the dynamic balance using the SEBT, a circle was assumed on the ground, and 8 lines were drawn at a 45-degree angle (like a star). The patient was asked to stand on the affected leg in the center of the circle and move the other leg as far as possible on the lines (in the selected direction), touch the ground with the toe at maximum reach without maintaining weight, and then return to the starting position (standing on two legs). The distance between the center of the star and the point of contact of the contralateral leg was considered the reach distance, an indicator of dynamic balance. This test was repeated three times, and their average was calculated and determined as the final score. The errors that cause exclusion from the test were displacement of the leg placed in the star center, loss of balance during the test, and putting weight on the reaching leg when the toe was in contact with the ground [18]. In previous studies, 
the reproducibility and high validity of these scales have been reported to evaluate the outcomes in patients with ACL reconstruction [19-23].

All participants were evaluated in the first stage of evaluation ( 2 weeks after ACL reconstruction surgery) and again in the second stage of evaluation (after 4 weeks of physiotherapy) using the mentioned instruments. In the last treatment session, the patients were asked to report changes in their health status from the beginning to the last session of treatment on a 7-point retrospective Likert scale [15, 24]. This scale was divided into two general subscales of improved (score 5-7) and not-improved (score 1-4) to create a twopart outcome variable [15]. Since the purpose of responsiveness studies is to evaluate the instrument's specificity rather than to evaluate the effectiveness of the intervention, the control of physiotherapy intervention was not necessary for the present study [24]. However, all patients received similar rehabilitation interventions after the surgery.

\section{Data analysis}

The obtained data were analyzed in SPSS v. 21 software. First, we used the Kolmogorov-Smirnov test to investigate the normal distribution of variables. The paired t-test was used to examine the relationship between pre- and posttreatment scores. The Receiver Operating Characteristics (ROC) curve (with 95\% confidence interval) was used to evaluate the responsiveness [25]. To do this, after two stages of evaluation, a change score (difference between the scores of two evaluation stages) was first obtained using the instruments for each patient. One by one, the change scores were determined as the cut-off point and compared with the score obtained from the 7-point Likert scale as a standard external criterion. Thus, sensitivity and specificity were calculated at each stage $[10,26,27]$. Then, the ROC curve was plotted, with the vertical axis corresponding to the sensitivity and the horizontal axis corresponding to the specificity [26]. In the ROC curve analysis, a point with the highest sensitivity and specificity was selected on the left and top of the curve, representing the minimum clinical change score of the outcome [14]. Besides, the correlation between the change score obtained from each instrument (as a quantitative variable) and the score obtained from the 7-point Likert scale (as a qualitative variable) were examined. The Spearman correlation coefficient in the range of $0.250-0.50$ indicates a weak correlation, 0.50-0.70 moderate correlation, and $>0.70$ indicates a strong correlation [10]. Similarly, the area below the ROC curve in the range of $0.25-0.50$ indicates poor responsiveness, $0.50-0.70$ shows moderate responsiveness, and $>0.70$ indicates good responsiveness [26].

\section{Results}

The demographic and clinical characteristics of participates are presented in Table 1, and the results of the paired t-test are presented in Table 2. As seen, 34 patients (63\%) were classified as an improved group, and 20 patients (37\%) as a not-improved group. The results obtained from the area below the ROC curve showed that the knee extension ROM, quadriceps and hamstring strength, and dynamic balance at medial and posteromedial directions had a good response $(<0.70)$. The Spearman correlation coefficient for these four outcomes between the change score and the score of 7-point global change rating Likert scale ranged from 0.36 to 0.51 . Significant clinical changes were reported for all study tools (Table 3 ).

\section{Discussion and Conclusion}

One of the most critical problems and complications after ACL reconstruction is the loss of knee extension end ROM (5-10 degrees) [28]. This ROM loss can lead to significant pain and functional impairment, especially during walking and running in young and active patients, because the loss of more than 10 degrees prevents normal gait and leads to increased loads on the patellofemoral joint and, as a result, anterior knee pain [28]. Loss of knee flexion end ROM is usually not as debilitating as loss of knee extension end ROM [28]. Therefore, changes in ROM in the direction of extension rather than in the direction of flexion are more consistent with changes perceived by the young patients, and this factor can be a reason for greater responsiveness of goniometer for assessing knee extension ROM. Another major problem after ACL reconstruction is the weakness of the quadriceps and hamstring muscles [29]. Several studies have shown a greater reduction in the quadriceps strength than in hamstring strength in these patients [29]. It has also been shown that the correlation between reduced quadriceps strength and knee function is higher than the correlation between reduced hamstring strength and knee function [30]. The results of our study also showed that the hand-held dynamometer's ability to respond to the knee extension strength (quadriceps muscle strength) was greater than the ability to respond to the knee flexion strength (hamstring muscle strength). However, it was reasonably responsive to both knee extension and flexion and can monitor the actual recovery of young and active ACL patients in clinical and research settings.

The results of the ROC curve and correlation analysis showed that VAS was not so much responsive to diagnose the ACL patient's real and significant recovery. In a systematic review and meta-analysis by Collins et al. on the measurement characteristics of the knee injury and osteoarthritis outcome score instrument in young patients with 
Table 1. Demographic and clinical characteristics of the participates $(n=54)$

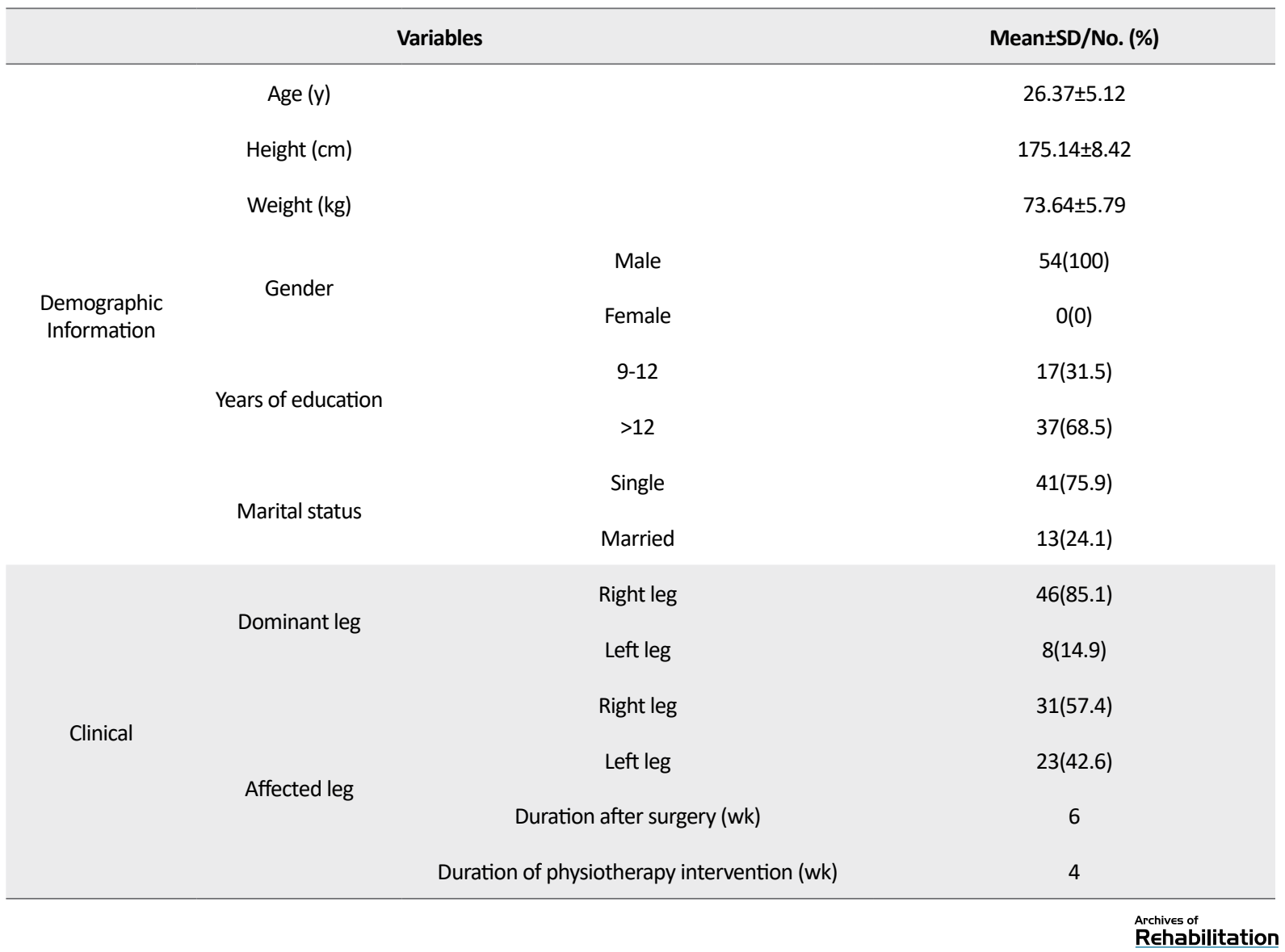

an ACL injury or knee osteoarthritis, it was shown that the pain subscale of the instrument had poor content validity and therefore had no good capacity to show improvement [31]. Consistent with this study, VAS in our study had the poor potential to show the changes in patients' real recovery after treatment, maybe, due to the participation of young and active patients. Another possible reason can be that the assessments were performed after the initial acute phase and in the intermediate phase of rehabilitation (2-6 weeks after surgery). During this period, patients typically have minimal pain and symptoms, and exercise during this period tends to be more difficult; hence, no significant change in pain is expected for ACL patients.

SEBT in medial and posteromedial directions showed acceptable responsiveness. The greater responsiveness in these two directions compared to other directions can be because these two directions exert more rotational force on the reconstructed knee during the test [32]. Thus, these two directions can more accurately identify important clinically significant changes in the dynamic balance of ACL patients. This result is somehow consistent with the findings of Herrington et al., who showed that the medial and pos- teromedial directions were more capable of differentiating between patients with ACL injury and controls [32]. The optimal cut-off point obtained from the ROC curve, which is considered as the least clinically significant change in the patient's health status, can provide researchers and therapists with practical and valuable information to make decisions about the actual change in ACL patient's health status.

The present study results provide evidence for the responsiveness of goniometer for knee extension ROM, hand-held dynamometer for quadriceps and hamstring strength, and SEBT for dynamic balance at medial and posteromedial directions in the assessment of clinical changes in patients with ACL reconstruction. The minimum clinically significant change score obtained for each tool in this study can help therapists and researchers to decide on determining the actual significant change in the ACL patient's clinical conditions after physiotherapy. 
Table 2. Mean scores of pre-treatment, post-treatment, and change for the study variables

\begin{tabular}{|c|c|c|c|c|c|}
\hline \multirow{2}{*}{ Variables } & & \multicolumn{3}{|c|}{ Mean \pm SD } & \multirow{2}{*}{$\mathbf{P}$} \\
\hline & & Pre-Treatment Score & Post-Treatment Score & Change & \\
\hline \multirow{3}{*}{ Pain } & Total $(n=54)$ & $2.83 \pm 1.71$ & $1.87 \pm 1.81$ & $0.96 \pm 1.80$ & $<0.001$ \\
\hline & Improved ( $n=34)$ & $2.65 \pm 1.45$ & $1.53 \pm 1.41$ & $1.11 \pm 1.83$ & 0.001 \\
\hline & Not improved $(n=20)$ & $3.15 \pm 2.08$ & $2.45 \pm 2.25$ & $0.70 \pm 1.75$ & 0.09 \\
\hline \multirow{3}{*}{ Knee flexion ROM } & Total $(n=54)$ & $117.86 \pm 11.34$ & $125.29 \pm 10.74$ & $8.40 \pm 7.75$ & $<0.001$ \\
\hline & Improved ( $n=34)$ & $119.44 \pm 10.28$ & $127.88 \pm 8.64$ & $8.37 \pm 7.47$ & $<0.001$ \\
\hline & Not improved $(n=20)$ & $115.16 \pm 12.78$ & $120.90 \pm 12.64$ & $8.46 \pm 8.28$ & 0.02 \\
\hline \multirow{3}{*}{ Knee extension ROM } & Total $(n=54)$ & $2.95 \pm 2.33$ & $1.01 \pm 1.56$ & $1.88 \pm 1.98$ & $<0.001$ \\
\hline & Improved $(n=34)$ & $3.65 \pm 1.85$ & $0.72 \pm 1.19$ & $2.80 \pm 1.53$ & $<0.001$ \\
\hline & Not improved $(n=20)$ & $1.78 \pm 2.63$ & $1.50 \pm 1.99$ & $0.30 \pm 1.66$ & 0.47 \\
\hline \multirow{3}{*}{ Quadriceps strength } & Total $(n=54)$ & $21.15 \pm 12.68$ & $25.73 \pm 12.02$ & $3.45 \pm 6.93$ & 0.004 \\
\hline & Improved ( $n=34$ ) & $21.91 \pm 12.89$ & $26.61 \pm 11.91$ & $5.31 \pm 14.00$ & 0.006 \\
\hline & Not improved $(n=20)$ & $19.86 \pm 12.54$ & $24.22 \pm 12.37$ & $0.29 \pm 9.47$ & 0.16 \\
\hline \multirow{3}{*}{ Hamstring strength } & Total $(n=54)$ & $10.92 \pm 6.89$ & $15.87 \pm 8.19$ & $5.44 \pm 10.95$ & $<0.001$ \\
\hline & Improved $(n=34)$ & $10.92 \pm 7.31$ & $15.76 \pm 8.52$ & $8.81 \pm 7.18$ & $<0.001$ \\
\hline & Not improved $(n=20)$ & $10.92 \pm 6.30$ & $16.06 \pm 7.82$ & $0.28 \pm 13.79$ & 0.01 \\
\hline \multirow{3}{*}{ Balance at anterior direction } & Total $(n=54)$ & $82.17 \pm 6.33$ & $88.19 \pm 7.22$ & $6.01 \pm 5.30$ & $<0.001$ \\
\hline & Improved $(n=34)$ & $81.96 \pm 6.82$ & $87.83 \pm 8.06$ & $5.87 \pm 5.76$ & $<0.001$ \\
\hline & Not improved $(n=20)$ & $82.55 \pm 5.55$ & $88.79 \pm 5.64$ & $6.25 \pm 4.54$ & $<0.001$ \\
\hline \multirow{3}{*}{ Balance at lateral direction } & Total $(n=54)$ & $59.70 \pm 11.31$ & $68.18 \pm 11.15$ & $8.47 \pm 6.41$ & $<0.001$ \\
\hline & Improved $(n=34)$ & $58.82 \pm 11.87$ & $67.69 \pm 11.62$ & $8.87 \pm 4.54$ & $<0.001$ \\
\hline & Not improved $(n=20)$ & $61.20 \pm 10.41$ & $69.01 \pm 10.55$ & $7.80 \pm 8.84$ & 0.001 \\
\hline \multirow{3}{*}{ Balance at medial direction } & Total $(n=54)$ & $71.06 \pm 9.75$ & $78.41 \pm 7.91$ & $7.35 \pm 8.85$ & $<0.001$ \\
\hline & Improved $(n=34)$ & $69.93 \pm 10.46$ & $78.83 \pm 8.98$ & $8.89 \pm 8.35$ & $<0.001$ \\
\hline & Not improved $(n=20)$ & $72.97 \pm 8.33$ & $77.69 \pm 5.80$ & $4.71 \pm 9.26$ & 0.03 \\
\hline \multirow{3}{*}{ Balance at posteromedial direction } & Total $(n=54)$ & $69.75 \pm 9.12$ & $76.29 \pm 9.52$ & $6.54 \pm 7.43$ & $<0.001$ \\
\hline & Improved $(n=34)$ & $67.74 \pm 9.04$ & $76.39 \pm 10.04$ & $8.65 \pm 6.26$ & $<0.001$ \\
\hline & Not improved $(n=20)$ & $73.17 \pm 8.40$ & $76.14 \pm 8.81$ & $2.96 \pm 8.04$ & 0.111 \\
\hline
\end{tabular}


Table 3. The Spearman correlation coefficient and area under the Receiver Operating Characteristics (ROC) curve for the study variables

\begin{tabular}{|c|c|c|c|c|c|}
\hline Variables & $\begin{array}{l}\text { The Spearman Correlation } \\
\text { Coefficient (P-Value) }\end{array}$ & $\begin{array}{l}\text { Area Under the Curve } \\
(95 \% \mathrm{Cl})\end{array}$ & Cut-Point & Sensitivity (95\% Cl) & Specificity $(95 \% \mathrm{Cl})$ \\
\hline Pain & $\begin{array}{l}0.16 \\
0.22\end{array}$ & $\begin{array}{c}0.61 \\
(0.45-0.76)\end{array}$ & 1.50 & $\begin{array}{c}0.52 \\
(0.35-0.69)\end{array}$ & $\begin{array}{c}0.85 \\
(0.61-0.96)\end{array}$ \\
\hline Knee flexion ROM & $\begin{array}{l}0.07 \\
0.57\end{array}$ & $\begin{array}{c}0.54 \\
(0.38-0.70)\end{array}$ & 8.83 & $\begin{array}{c}0.47 \\
(0.30-0.64)\end{array}$ & $\begin{array}{c}0.70 \\
(0.45-0.87)\end{array}$ \\
\hline Knee extension ROM & $\begin{array}{r}0.51 \\
<0.001\end{array}$ & $\begin{array}{c}0.85 \\
(0.73-0.97)\end{array}$ & 1.50 & $\begin{array}{c}0.82 \\
(0.64-0.92)\end{array}$ & $\begin{array}{c}0.85 \\
(0.61-0.96)\end{array}$ \\
\hline Quadriceps strength & $\begin{array}{r}0.41 \\
<0.001\end{array}$ & $\begin{array}{c}0.73 \\
(0.58-0.89)\end{array}$ & 1.52 & $\begin{array}{c}0.85 \\
(0.68-0.94)\end{array}$ & $\begin{array}{c}0.65 \\
(0.40-0.83)\end{array}$ \\
\hline Hamstring strength & $\begin{array}{l}0.36 \\
0.002\end{array}$ & $\begin{array}{c}0.70 \\
(0.54-0.86)\end{array}$ & 1.77 & $\begin{array}{c}0.82 \\
(0.64-0.92)\end{array}$ & $\begin{array}{c}0.60 \\
(0.36-0.80)\end{array}$ \\
\hline $\begin{array}{l}\text { Reach at anterior } \\
\text { direction }(\mathrm{cm})\end{array}$ & $\begin{array}{l}-0.01 \\
0.90\end{array}$ & $\begin{array}{c}0.45 \\
(0.29-0.60)\end{array}$ & 10.91 & $\begin{array}{c}0.26 \\
(0.13-0.44)\end{array}$ & $\begin{array}{c}0.90 \\
(0.66-0.98)\end{array}$ \\
\hline $\begin{array}{l}\text { Balance at lateral } \\
\text { direction }(\mathrm{cm})\end{array}$ & $\begin{array}{l}0.12 \\
0.33\end{array}$ & $\begin{array}{c}0.56 \\
(0.40-0.72)\end{array}$ & 10.50 & $\begin{array}{c}0.41 \\
(0.25-0.59)\end{array}$ & $\begin{array}{c}0.85 \\
(0.61-0.96)\end{array}$ \\
\hline $\begin{array}{l}\text { Balance at medial } \\
\text { direction }(\mathrm{cm})\end{array}$ & $\begin{array}{l}0.38 \\
0.001\end{array}$ & $\begin{array}{c}0.71 \\
(0.56-0.86)\end{array}$ & 5.08 & $\begin{array}{c}0.73 \\
(0.55-0.86)\end{array}$ & $\begin{array}{c}0.70 \\
(0.45-0.87)\end{array}$ \\
\hline $\begin{array}{l}\text { Balance at posterome- } \\
\text { dial direction }(\mathrm{cm})\end{array}$ & $\begin{array}{c}0.43 \\
<0.001\end{array}$ & $\begin{array}{c}0.74 \\
(0.59-0.88)\end{array}$ & 4.25 & $\begin{array}{c}0.73 \\
(0.55-0.86)\end{array}$ & $\begin{array}{c}0.65 \\
(0.40-0.83)\end{array}$ \\
\hline
\end{tabular}

Archives of

\section{Ethical Considerations}

\section{Compliance with ethical guidelines}

All ethical principles are considered in this article. The participants were informed about the purpose of the research and its implementation stages. They were also assured about the confidentiality of their information and were free to leave the study whenever they wished, and if desired, the research results would be available to them. was approved by the Ethics Committee of Ahvaz Jundishapur University of Medical Sciences (Code: IR.AJUMS. REC.1394.275).

Funding

This study was extracted from a PhD. dissertation of the first author at the Musculoskeletal Rehabilitation Research Center, Ahvaz
University of Medical Sciences, Ahvaz. Also, this study was supported by the Ahvaz Jundishapur University of Medical Sciences.

Authors' contributions

Conceptualization: Mohammad Jafar Shaterzadeh Yazdi, Hossein Negahban, Neda Mostafaee; Supervision: Mohammad Jafar Shaterzadeh Yazdi, Hossein Negahban, Shahin Goharpey; Methodology: Hossein Negahban, Shahin Goharpey, Neda Mostafaee; Writing - original draft: Neda Mostafaee; Writing - review \& editing: All authors; Data collection: Neda Mostafaee, Nahid Pirayeh; Data analysis: Mohammad Mehravar, Neda Mostafaee.

\section{Conflict of interest}

The authors declared no conflict of interest.

Acknowledgments 
This Page Intentionally Left Blank 
مقاله بزخوهشى:

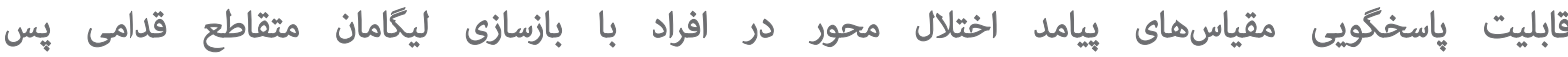
از فيزيوترايى تئي

ندا مصطفايى' •، حسين نكهبان"، محمدجعفر شاطرزاده يزدى"، شاهين كوهريى"، محمد مهرآور"، "ناهيد بيرايه

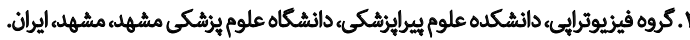

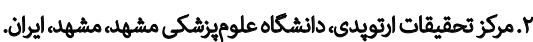

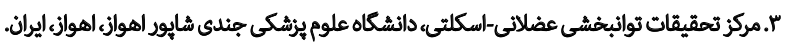

\begin{abstract}
حكبد

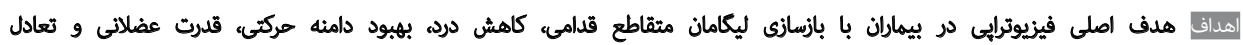

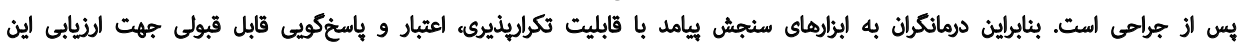

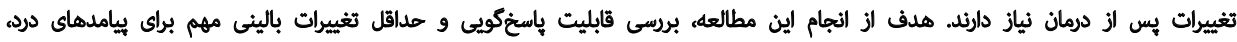

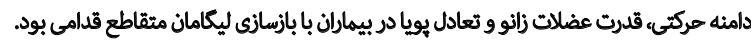

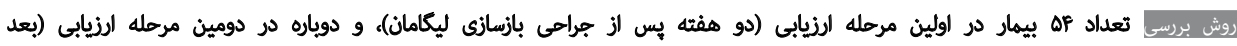

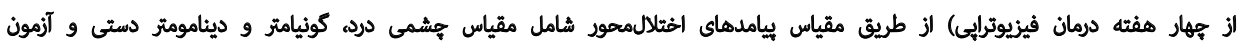

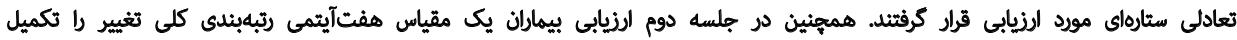

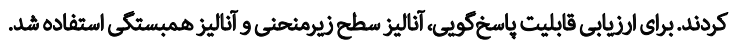

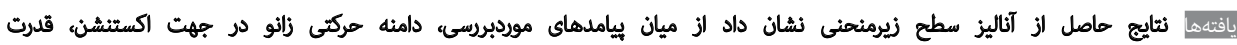

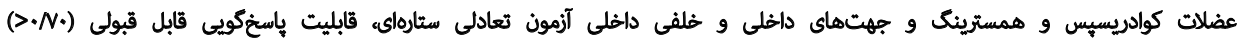

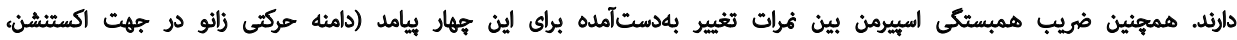

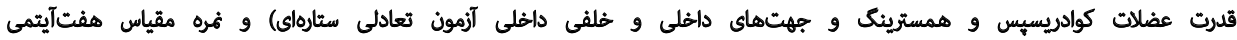

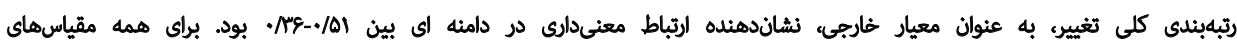

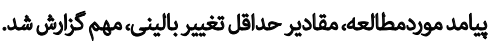

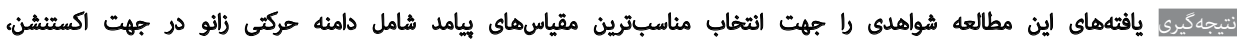

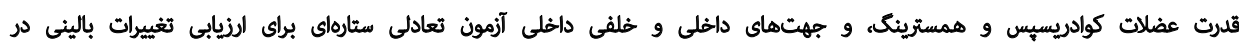

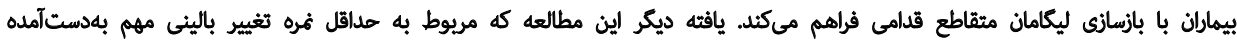

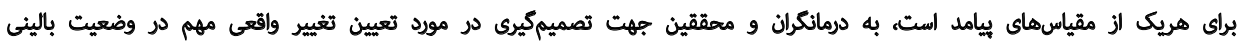

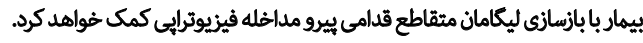

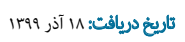
تاريغ بليرش: م· بهمن

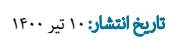

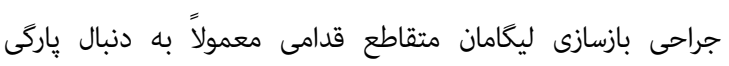

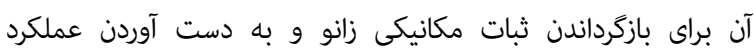

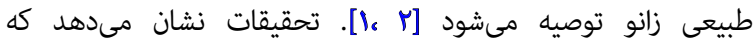

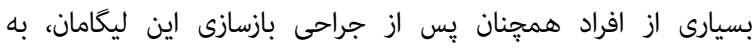

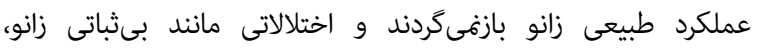
درد، كاهش دامنه حركتى و نقص در قدرت عضلات كوادريسيس و و ونيس

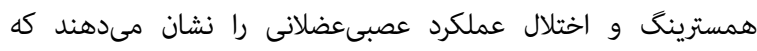

ياركى ليكامان متقاطع قدامى يكى از شايعترين آسيبهاى

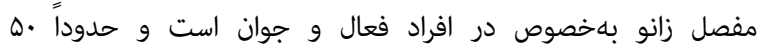

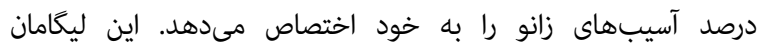

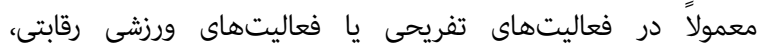

دجار آسيب مىشود [1]].

نويسنده مسئول:

ناهيد ييرايه

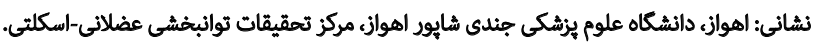

تلفن:

رايانامه: nahid_pt8287@yahoo.com 
اندازهگيرى مرجع رخ داده است. منظور از اندازهكيرى مرجع، مقياس ليكرت هفترتبهاى كذشتهنكر است [عآ]ت. علىرغم اينكه

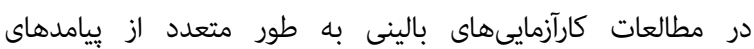

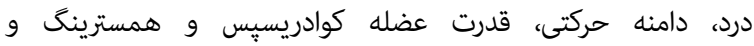

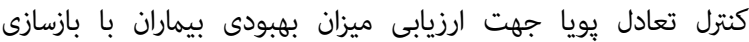

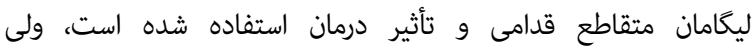

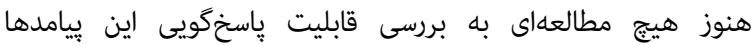

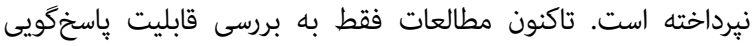

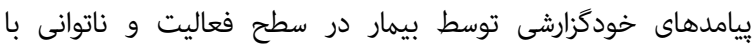

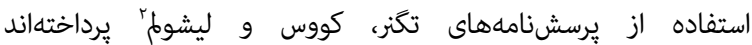

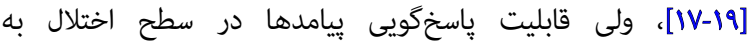

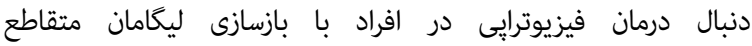

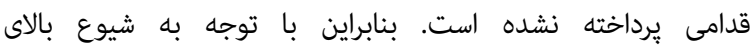

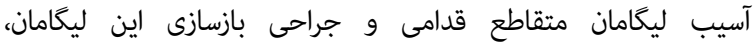

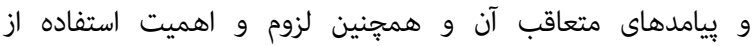

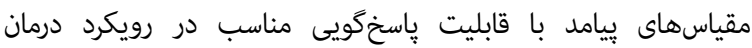

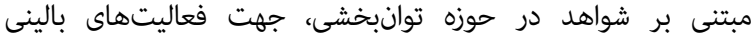

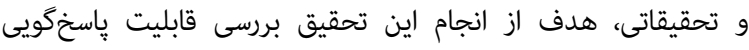

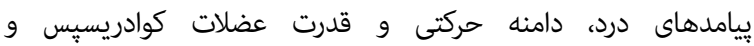

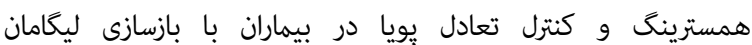
متقاطع قدامى متعاقب درمان توانبخشى است.

روش بررسى

شركت كنندهما

اين يزوهش به صورت همخروهى آيندهنكر انجام شد. در اين

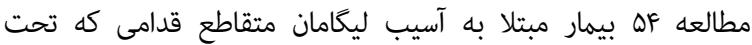

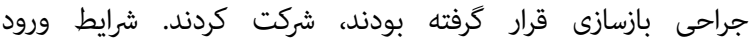

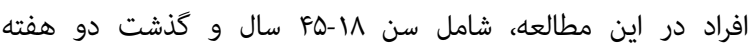

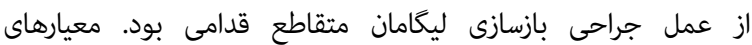

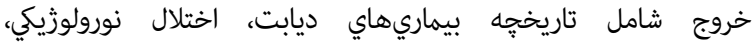

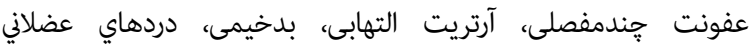

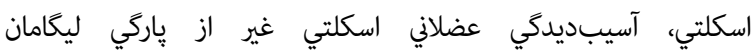

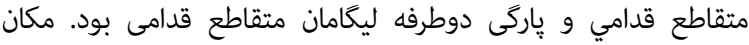

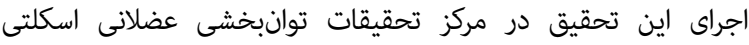

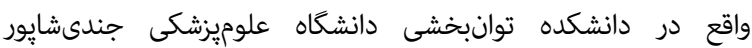

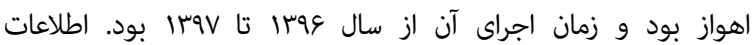

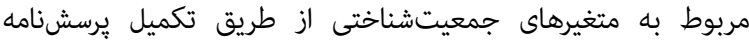
توسط خود بيمار جمع آورى شد.

براى ارزيابى ييامد درد از مقياس قياسى جشمى VAS' استفاده

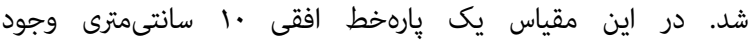

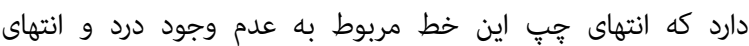

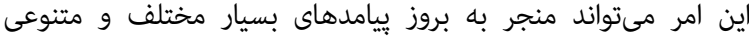

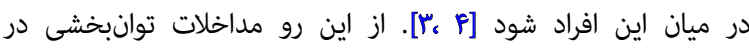

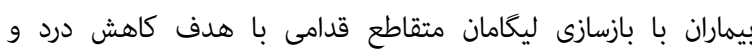

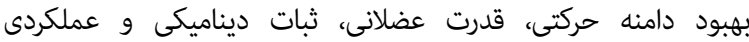

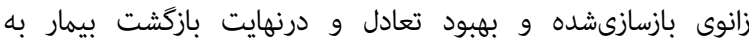
فعاليتهاى عملكردى ضرورى است [ه].]

بر اساس مطالعات مرورى سيستماتيك، از جمله رايجترين

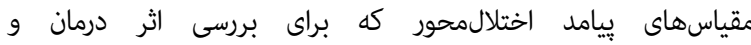

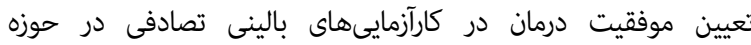

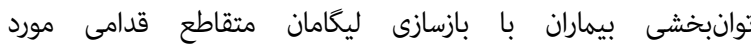

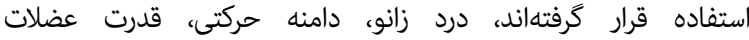

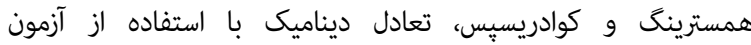

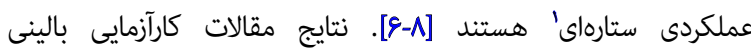

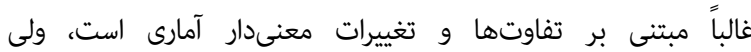

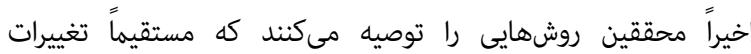

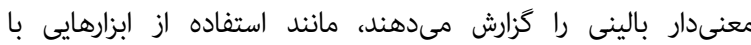

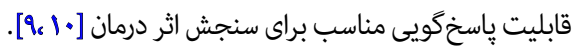
ممكن است يك ابزار تكراريذير و معتبر باشد، ولى قادر به

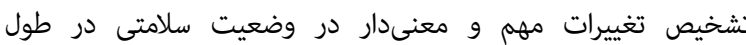

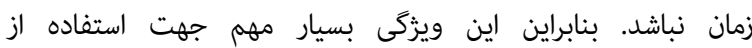

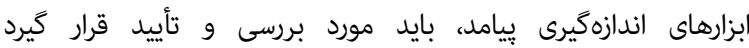

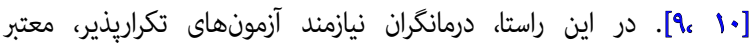

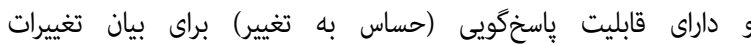

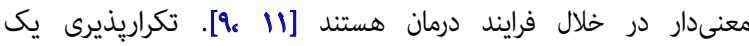
ابزار به معنى آن است كه نمرات يك ابزار سنجش تا جه مهار ميزان

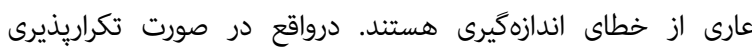

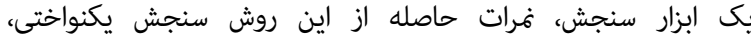

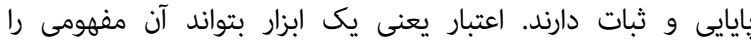

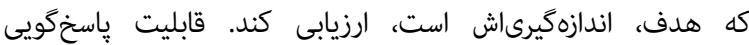

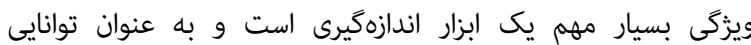

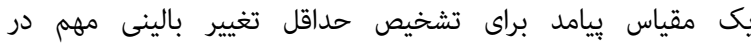

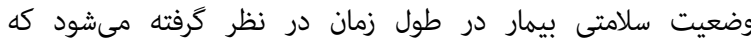

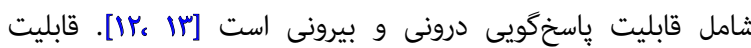

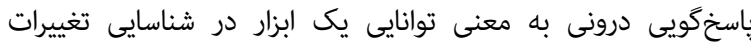

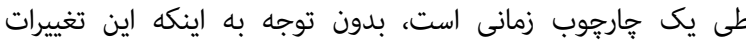

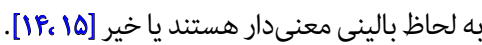

قابليت ياسخكويى درونى اغلب به وسيله استفاده از ابزار قبل و بعد از يك برنامه درمانى مطمئن كه اثر درمانى آن به طور كامل بله

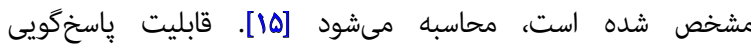
بيرونى نشاندهنده آن است كه در يك مقاسيه مقطع زمانى مشخص، جه ميزان تغيير در ابزار اندازمگيرى مطابق با تغيير در ابزار 


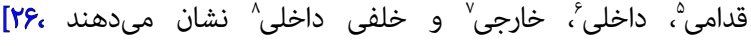

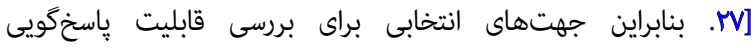

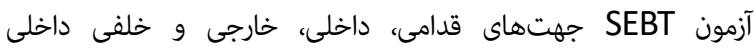

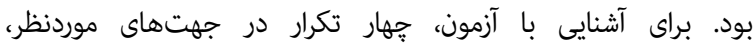

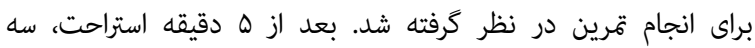

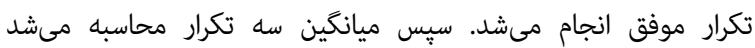

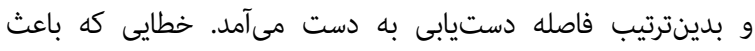

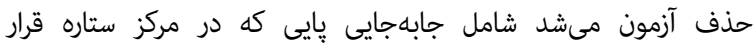

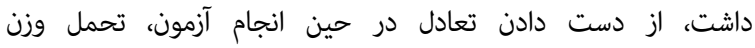

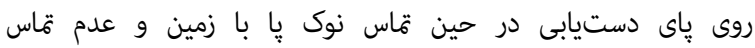

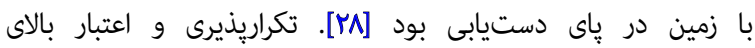

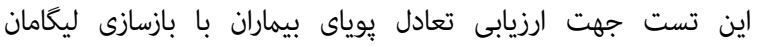

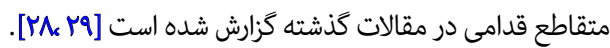

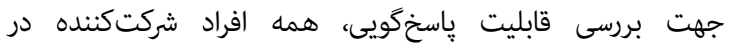

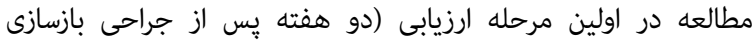

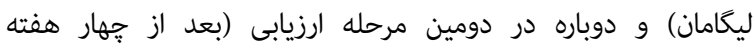

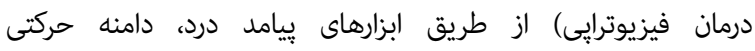

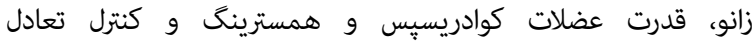

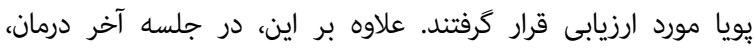

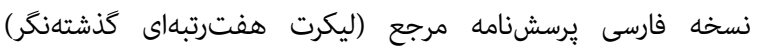

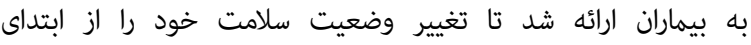

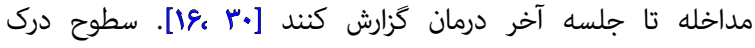

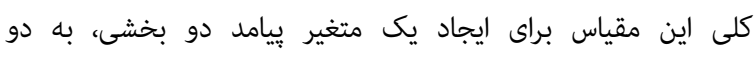

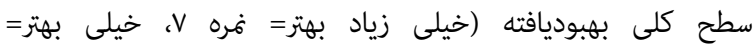

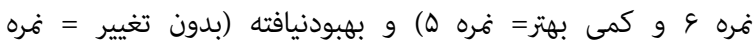

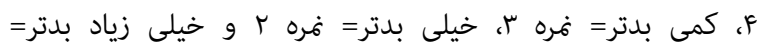

فره ال ) تقسيم بندى شد [19].

از آنجايى كه هدف از مطالعات قابليت ياسخكويى، بررسى

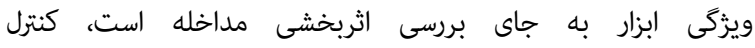

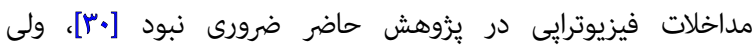

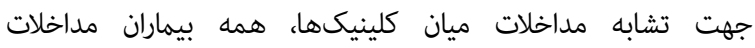

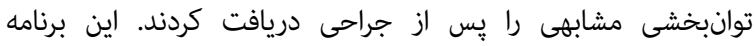

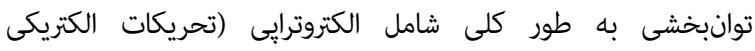

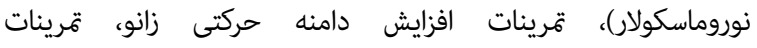

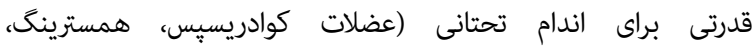

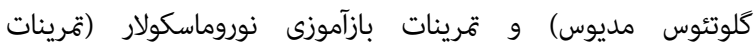

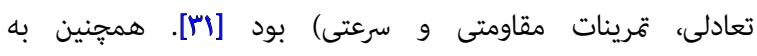

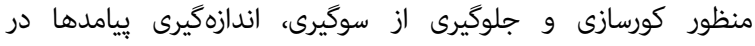
زمان قبل و بعد از درمان توسط فردى غير از درمانكر انجام شد.

\section{Anterior}

6. Medial

7. Lateral

8. Posterior-medial
ديكر آن مربوط به شديدترين دردى است كه فرد تجربه مىكند.

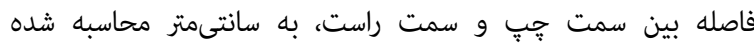

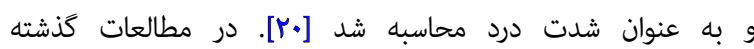

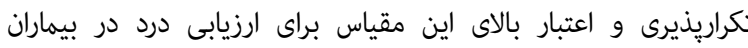

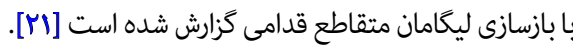

از كونيامتر براى اندازهكيرى دامنه حركتى ياسيو زانو در در

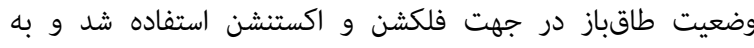

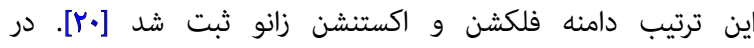

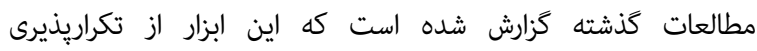

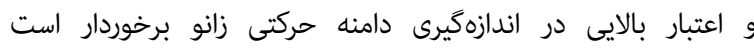

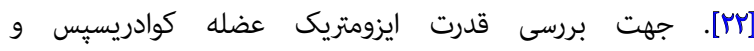
همسترينگ از دينامومتر دستى كششى فشارى (ساخت شرئ شركت

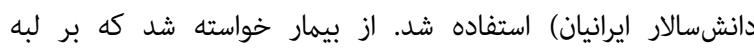

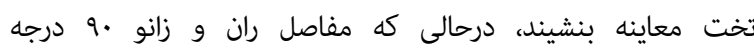

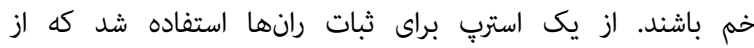

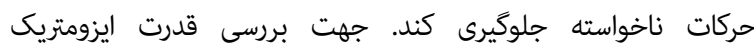

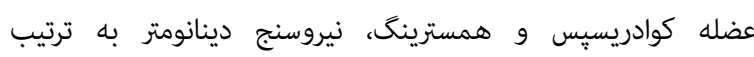
در بخش قدامى و خلفى ساق، درست بالاى مالئولها قرار كرّفت.

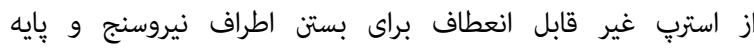

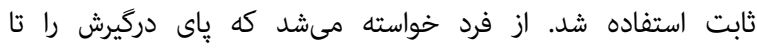

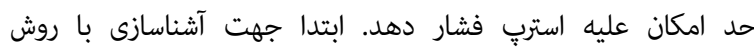

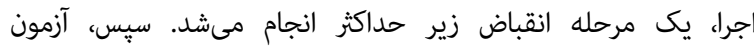

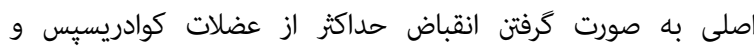

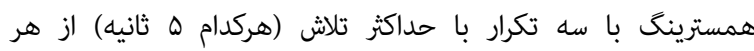

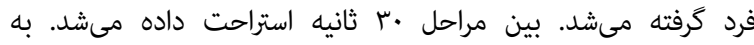

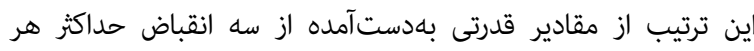

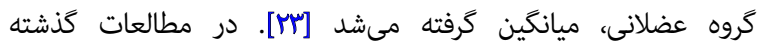

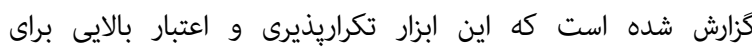

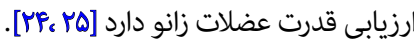

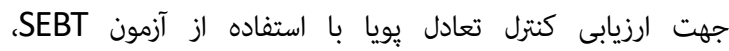

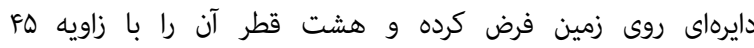

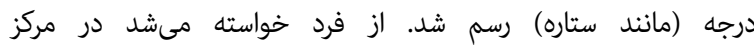

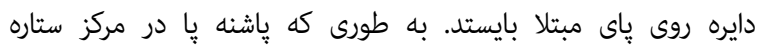

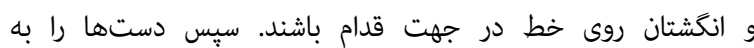

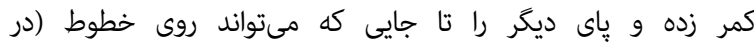

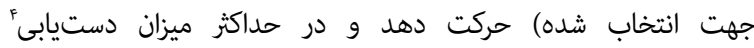

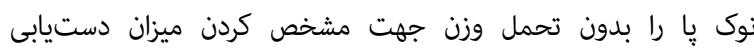

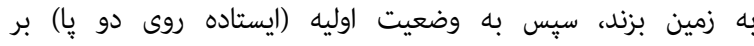

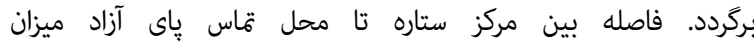

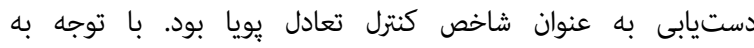

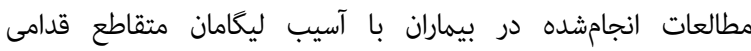
ديده شده كه اين بيماران اختلالات عملكردى را در جهتهاريان

\section{Reach}


مثبت صحيح قرار دارند، نسبت به كَروهى از بيماران كه طبق

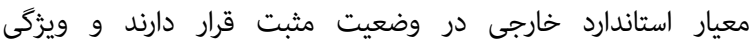

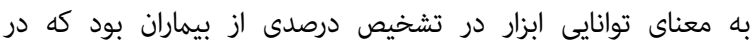

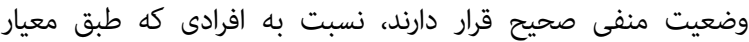

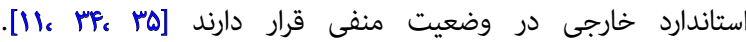

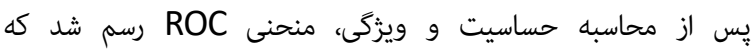

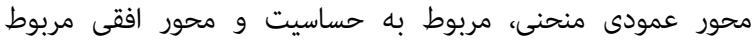

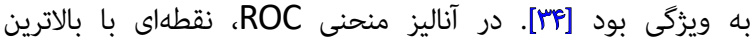

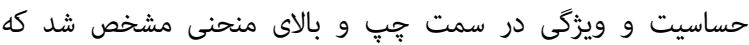

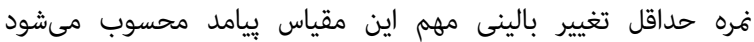

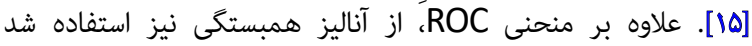

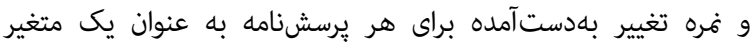

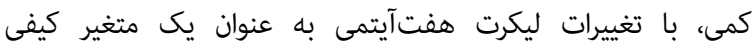

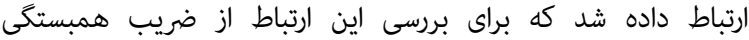

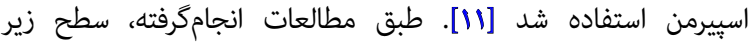

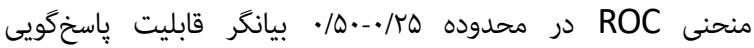

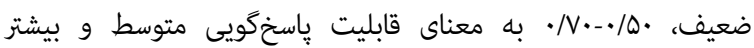

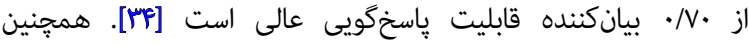
ضريب همبستكى اسييرمن در محدوده

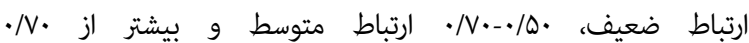

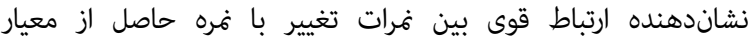

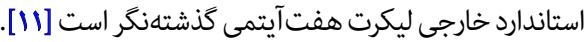

يافتهها

مشخصات جمعيتشناختى و بالينى بيماران شركتكننده در جدول شماره ا آمده است. نتايج آمار توصيفى و ميزاني

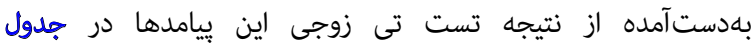

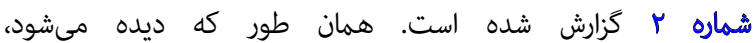

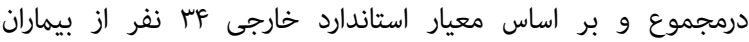

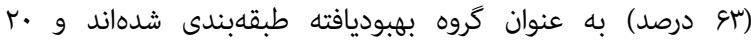
نفر از بيماران (rVV درصد) در دسته بيماران بهبوديونيافته قرار

نتايج بهدستآمده از سطح زير منحنى ROC نشان داد از ميان

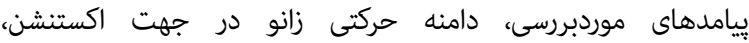

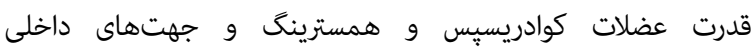

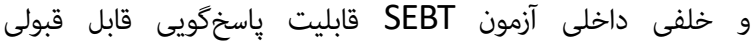

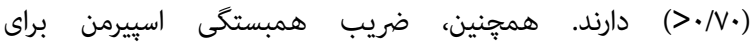

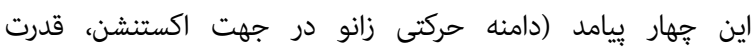

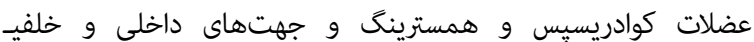

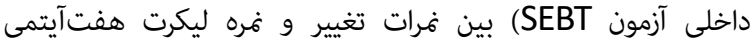

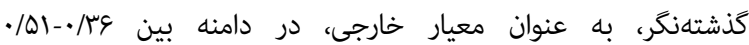
بود. براى همه مقياسهاى ييامدهاى مورد مطالعه، مقادير حدانئ حداقل

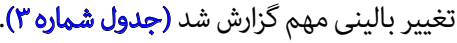

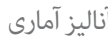

جهت بررسى توزيع نرمال متغيرها، از آزمون كوطوكروف اسميرنوف استفاده شد. از آزمون تى زوجى نيز براى بررسى برى

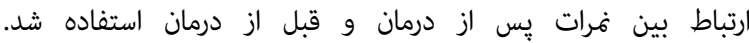
درنهايت دادهها در نرمافزار SPSS نسخه الب مورد تجزيه و تحر تحليل

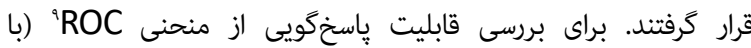

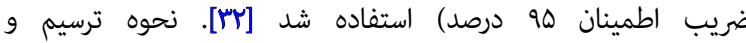

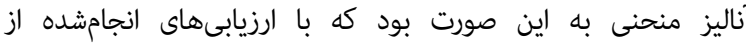

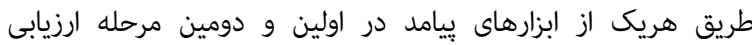
فرهاى به دست مى آمد و بعد از دو مرحله ارزيابى براى هر إنائ

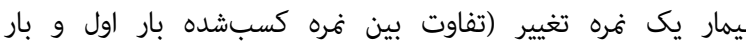

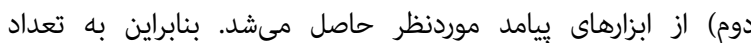
بيماران حاضر در يزوهش نهره تغيير وجود داشت. از طرفى بيمار

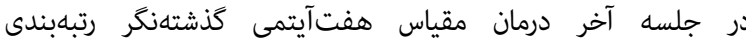
ليكرت را دريافت و تكميل مىكرد [بست].

سطوح درى كلى اين مقياس براى ايجاد يك متغير يِيامد

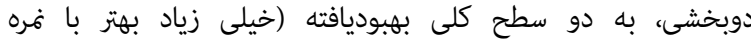

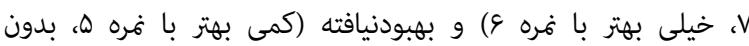

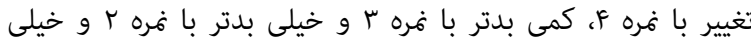

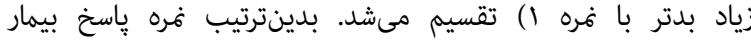

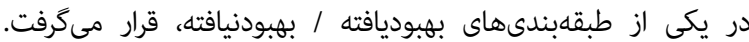

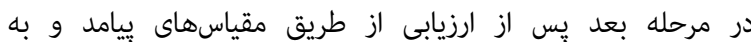

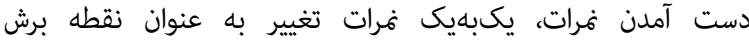

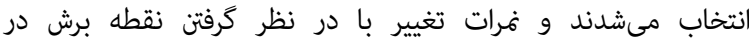

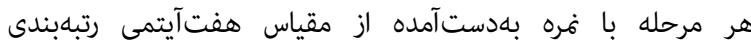

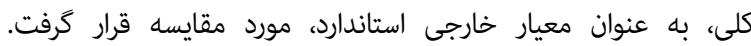

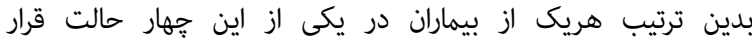

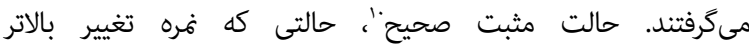
از نقطه برش باشد و در ليكرت هفتآيتمى هم بهبت هاتيودى كزارش

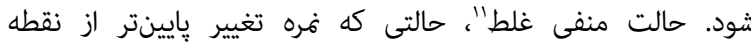

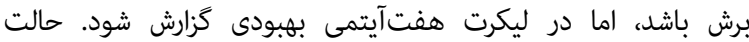

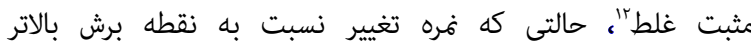

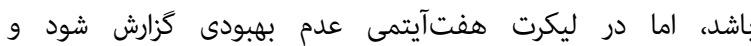

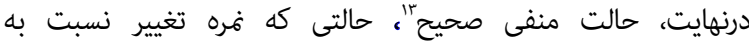

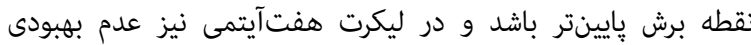

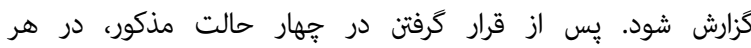

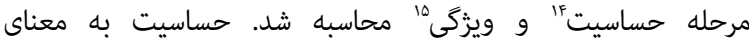
توانايى ابزار در تشخيص درصدى از بيماران بود كه در در وضعيت

\section{Receiver Operating Characteristics}

10. True positive

11. False negative

12. False positive

13. True negative

14. Sensitivity

15. Specificity 


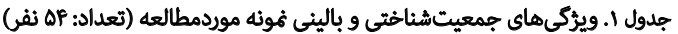

\begin{tabular}{|c|c|c|c|}
\hline تعلداد (درصد) & & هتغيرهاي دموكرافيك و باليثى & \\
\hline$r g / T V(\Delta / M)$ & & سن (سال)، ميانكين (اتحرافى معيان) & \\
\hline IVO/IF(N/PY) & & قد (سانتىهت)، ميانكين (انحراف معيار) & \\
\hline$V \Psi / 9 \%(\Delta / N q)$ & & وزن (كيلوكرم)، ميانكين (انحراف معيار) & \\
\hline \multirow[t]{2}{*}{$\Delta F(1 \cdot . / \cdot)$} & مرد & & \\
\hline & & جنسيت & \\
\hline.$(\%)$ & is & & ويزّكى هاى جمعيتشناختى \\
\hline \multirow[t]{2}{*}{$\operatorname{IV}(\mathrm{r} / \mathrm{Q})$} & $9-14$ & & \\
\hline & & سطح تعصيلات & \\
\hline$r V(\& N A)$ & $>i r$ & & \\
\hline \multirow[t]{2}{*}{$P(V 0 / q)$} & مجرد & & \\
\hline & & وضعيت تأهل & \\
\hline $\mathbb{H}(Y F / 1)$ & 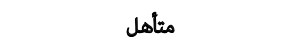 & & \\
\hline \multirow[t]{2}{*}{$P f(\Lambda \Delta / 1)$} & 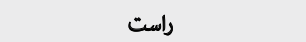 & & \\
\hline & & ل ياي غالب & \\
\hline$\wedge(1 F / 9)$ & جهب & & \\
\hline \multirow[t]{2}{*}{$M(\Delta V / \uparrow)$} & 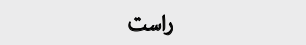 & & \\
\hline & & & اطلاعات بالينى \\
\hline \multirow[t]{2}{*}{$r(P r / q)$} & جيب & & \\
\hline & & ياى جراحىشده & \\
\hline 8 & مدتزمان يس از جراهمى (هفته) & & \\
\hline f & مدتزمان مداخله فيزيوترايى (هفته) & & \\
\hline
\end{tabular}

به نظر مىرسد كه تغييرات در دامنه حركتى در جهت اكستنشن نسبت به جهت فلكشن، با توجه به سطح فعاليت بالاى بيماران

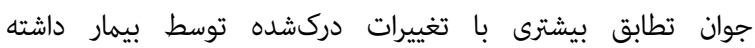

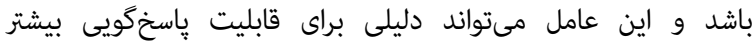

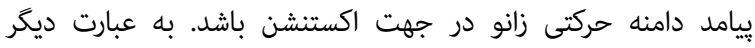

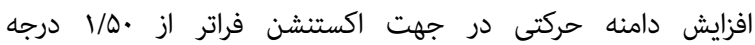
مىتواند فايانكر دقيقتر و مناسبترى بردئ دراى بهبودى واقعى بيمار

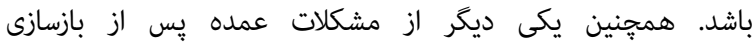

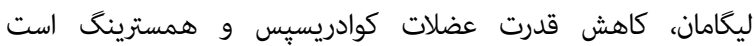

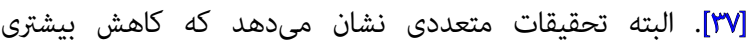

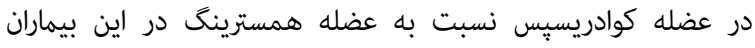

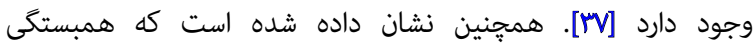

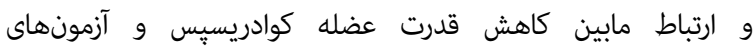
عملكردى زانو نسبت به همبستكى مابين كاهش قدرت فرت عضله

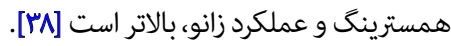

نتايج اين مطالعه نيز نشان مىدهد كه ميزان قابليت پاسخكويى

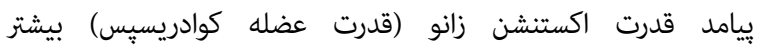

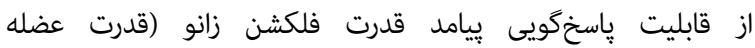

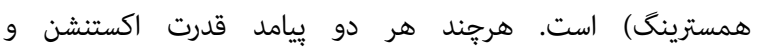

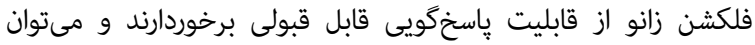

\section{بحث}

با توجه به بررسى مطالعات انجامشده تاكنون، اين مطالعه

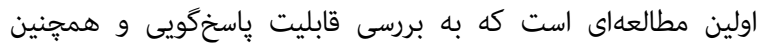

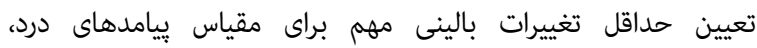

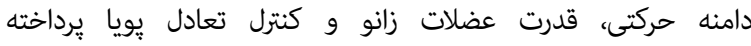
است. نتايج اين مطالعه مىتواند اطلاعات مفيدى را براى انتخاب

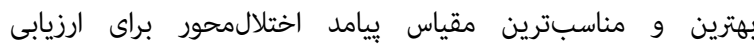
تغييرات بالينى، متعاقب درمان فيزيوترايى در اختيار درمانكران و

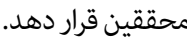

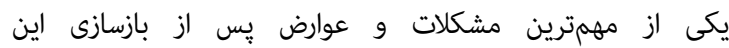

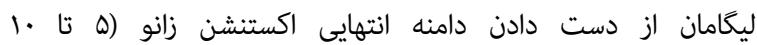

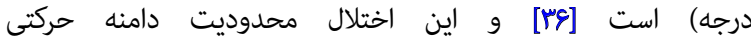
مىتواند منجر به درد قابل توجه و نقص عملكردى بهويزه در

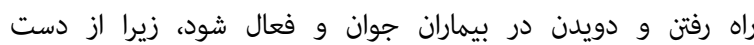

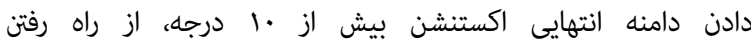

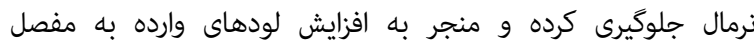

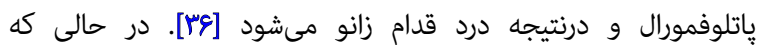

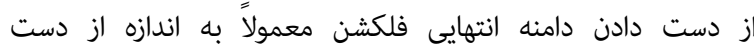

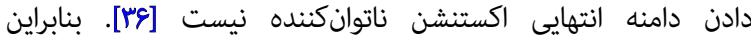


جدول r. ميائكين و أتعراف معيار نهرات اوليه (اولين جلسه ارزيابى)، ثائويه (دومين جلسه ارزيابى و نمرات تغيير براى ييامدهاى درد، دامنه حركتى، قدرث عضلات SEBT كانو و آثمون

\begin{tabular}{|c|c|c|c|c|c|}
\hline \multirow{2}{*}{$\mathbf{P}$} & \multicolumn{3}{|c|}{ ميانكين土|نحراث معيار } & \multirow{2}{*}{\multicolumn{2}{|c|}{ ت بيامدها }} \\
\hline & تغيير & فرات بعلد از درمان (دومين جلسه ارزيابى) & نمرات اوليه (اولين جلسيه ارزيابى) & & \\
\hline$<* / *+1$ & $1 / A \cdot \pm \cdot / 98$ & $V / A V \pm \backslash / A I$ & $r / A r \pm I / N$ & مجموع (n=AF) & \\
\hline $.1 \cdot+1$ & $1 / A r \pm V / 11$ & $1 / \Delta r \pm V / F I$ & $r / F \Delta \pm 1 / F \Delta$ & بهبوديافته (n=rf) & درد \\
\hline$\therefore / .9$ & INa土.N. & $r / F O \pm r / T O$ & $r / 1 \Delta \pm r / \cdot \Lambda$ & بهبودنياقته & \\
\hline$<\cdot 1 \cdot .1$ & VND士NP. & IrQ/TSEI.NP & IIV/NE士 I/NF & $(n=\Delta P) \varepsilon$ & \\
\hline$<+1+\cdot 1$ & $V / F V \pm N / T V$ & $I T V / M \pm N F F$ & $11 V / F+ \pm 1 \cdot / T \Lambda$ & (n=me) بهبودياوتئ & دامنه حركتى فلكشن \\
\hline .1 .4 & NTA土NFF & $\| r \cdot / q \cdot \pm I r / g p$ & $118 / 18 \pm 1 Y / N A$ & بهيودنياقته (n=r. به & \\
\hline$<+/++1$ & $1 / Q 4 \pm 1 / M$ & $y \cdot 1 \pm v / \Delta s$ & $r / q \Delta \pm Y / T$ & مجهموع (n=ه*) & \\
\hline$<* / * 1$ & $1 / \Delta r \pm r / A$ & $\cdot M+1 / 19$ & $r / F \Delta \pm V / \wedge \Delta$ & 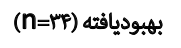 & دامنه حركتى اكستنشن \\
\hline.$/ F V$ & $1 / 89 \pm \cdot / \%$ & $1 / 0 \cdot \pm 1 / 99$ & INA土r/RT & بهبودنياثته & \\
\hline$\%+\%$ & $9 / 9+T / F \Delta$ & $r \Delta / M+ \pm I r / \cdot r$ & rI/IQEIr/gn & مجهوع (n=Ak) & \\
\hline$\%$ & $\mid F / \cdot \pm \pm \Delta /$ & r $/ 91 \pm 1 / 91$ & 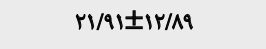 & 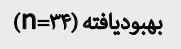 & قدرت عضله كوادريسيس \\
\hline.$/ 18$ & १/FV士./พจ & TE/TYIIT/NV & 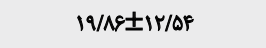 & بهبودنياقته (n=r. & \\
\hline$<+*+1$ & $1+/ 9 \Delta \pm \Delta / \% \varphi$ & $10 / / V \pm N / 19$ & $1 \cdot / 4 r \pm \xi / \wedge q$ & مجموع (n=AH) & \\
\hline$<\cdot 1 \cdot \cdot \cdot 1$ & $V / M A N / A I$ & $10 / N F \pm N A r$ & $1 . / 4+ \pm V / m$ & (n=RF) بهبوديافته & قدرث عضله همسترينك \\
\hline .1 .1 & IrNA土./TA & $19 / .9 \pm V / A r$ & 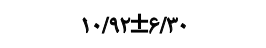 & بهبودنيافته & \\
\hline$<+/++1$ & $\Delta / r+ \pm s / \cdot 1$ & M/A $\pm V / r Y r$ & Ar/IV $\pm g / \pi r$ & هجموع (n=AF) & \\
\hline$<\bullet / . \cdot 1$ & $\Delta / V F \pm \Delta / A V$ & $\Lambda V / A N \pm N \cdot \varphi$ & AV/R $\pm \& / A r$ & بهبوديائته (n=FF) & جهت قدامى آزمون SEBT \\
\hline$<\cdot 1 \cdot .1$ & $P / O F \pm P / T Q$ & MVq土D/9P & $A Y / \Delta \Delta \pm \Delta / \Delta \Delta$ & بهبودنيافته (n=r. & \\
\hline$<+,+\infty 1$ & G/PIDN/FV & GNM & $\Delta q / N \cdot \pm 11 / m$ & (n=هF) & \\
\hline$<\bullet / \bullet \cdot 1$ & $P / O F \pm N A V$ & eV/R9 $\pm 11 / 8 T$ & QNANIIV/AV & بهبوديافتيه (n=rip & جهت خارجى آزيون SEBT \\
\hline.$/ . .1$ & NAFIV/A. & $99 / \cdot 1 \pm 1 \cdot 100$ & $81 / \pi \cdot \pm 1 \cdot / \% 1$ & بهبيودنيافتث (n=r. & \\
\hline$<+/ \cdot+1$ & $N \wedge \Delta \pm V / r \Delta$ & $V / / F I \pm V / q 1$ & $\mathrm{~W} / \cdot \odot \pm \mathrm{V} / \mathrm{B}$ & (n=AF) & \\
\hline$<\cdot / \cdot \bullet 1$ & NYMINA & VNAFIN/R & ร9/१ษ $\pm 1 . / \%$ & 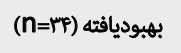 & جهت داخلى آزمون SEBT \\
\hline.$/ .4$ & Q/TE士FM & $W / 89 \pm \Delta / A$. & VK/RVINTT & 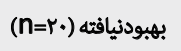 & \\
\hline$<+/ * 1$ & $V / F T E / \Delta F$ & $v g / r q \pm v / \Delta r$ & sqNatv/r & مجموع (n=AF) & \\
\hline$<\cdot 1 \cdot \cdot 1$ & P/KY士NFO & $v g / r q \pm 1 . \%$ & SVNF士Q/.F & بهيوديافته (n=Fif & جهت خلفى داخلى آزمون \\
\hline.$/ 111$ & $N \cdot P \pm r / q F$ & $V E / I I \pm N A I$ & $V \sim / V \pm N F$. & بهبودنيافنه (n=r. & \\
\hline
\end{tabular}


جدول r. ضريب همبستّى اسييرمن و سطح زير منحنى براي بيامدهاى درده دامنه حركتى و قدرث عضلات زائو و آزمون SEBT بر اساس معيار دوبخشى از مقياس استاندارد خارجى رتبهبندي كلى تغيير

\begin{tabular}{|c|c|c|c|c|c|}
\hline 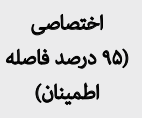 & (10 دروصد فاصله & تخططه برش هطلوب & 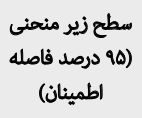 & 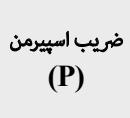 & ي ييامدها \\
\hline $\begin{array}{c}. / 1 \Delta \\
(\cdot / 91-. / 99)\end{array}$ & $\begin{array}{c}. / \Delta T \\
(. / \% 0-. / 89)\end{array}$ & $1 / 0$. & $\begin{array}{c}. / 91 \\
(\cdot / F \theta-. / 99)\end{array}$ & $\begin{array}{l}. / 19 \\
(\cdot / N Y)\end{array}$ & درد \\
\hline $\begin{array}{c}\cdot N \cdot \\
(\cdot / F \Delta-\cdot / A V)\end{array}$ & $\begin{array}{c}\cdot / F V \\
(\cdot / \pi \cdot-\cdot / q A)\end{array}$ & NAT & $\begin{array}{c}\cdot / \Delta P \\
(\cdot / \Gamma \Lambda-\cdot / N \cdot)\end{array}$ & $\begin{array}{l}\cdot / \cdot v \\
(\cdot / 0 V)\end{array}$ & دامثله حركتى فلكشئ \\
\hline $\begin{array}{c}. / 10 \\
(\cdot / 41-. / 99)\end{array}$ & $\begin{array}{c}\cdot / \mathrm{Ar} \\
(\cdot / \& 4-. / Q Y)\end{array}$ & $1 / 0$. & $\begin{array}{c}\cdot / \wedge \Delta \\
\left(\cdot / N^{w-} \cdot / q V\right)\end{array}$ & $\begin{array}{c}. / 01 \\
(<+/++1)\end{array}$ & دامنه حركتى اكستشن \\
\hline $\begin{array}{c}\cdot / \& \Delta \\
(\cdot / \% \cdot-\cdot / A r)\end{array}$ & $\begin{array}{c}\cdot / / A \\
(\cdot / 8 \Lambda-\cdot / q 4)\end{array}$ & Var & $\begin{array}{c}\cdot N{ }^{W} \\
(\cdot / \Delta \Lambda-\cdot / \wedge 9)\end{array}$ & $\begin{array}{c}. / 91 \\
(<\cdot / . .1)\end{array}$ & قدرت عضله كوادريسيس \\
\hline $\begin{array}{c}\cdot / 8 \cdot \\
(\cdot / \%-\bullet / 4 \cdot)\end{array}$ & $\begin{array}{c}. / A T \\
(. / 9 P-. / Q Y)\end{array}$ & $1 / W$ & $\begin{array}{c}\cdot N \cdot \\
(\cdot / \Delta P-\cdots / N \ominus)\end{array}$ & $\begin{array}{c}. / \% 8 \\
(\cdot . r / \cdot)\end{array}$ & قدرت عضله همسترينك \\
\hline $\begin{array}{c}. / 9 . \\
(\cdot / 98-\cdot / 4)\end{array}$ & $\begin{array}{c}\cdot / F E \\
(\cdot / / F-\cdot / F F)\end{array}$ & $1 . / 91$ & $\begin{array}{c}+/ F Q \\
(. / \% q-\circ / 8 \cdot)\end{array}$ & $\begin{array}{l}-. / .1 \\
(. / 9.9)\end{array}$ & جهت قدامى آزمون SEBT (ساثتىمتر) \\
\hline $\begin{array}{c}\cdot / \wedge \Delta \\
(\cdot / 91-\cdot / q 9)\end{array}$ & $\begin{array}{c}\cdot / P 1 \\
(\cdot / r \Delta-. / \Delta q)\end{array}$ & $1 . / \Delta *$ & $\begin{array}{c}\cdot / \Delta F \\
(\cdot / F \cdot \ldots / N r)\end{array}$ & $\begin{array}{l}\cdot / r \\
(\cdot / T)\end{array}$ & جهت خارجى آزمون SEBT (سانتىمثي) \\
\hline $\begin{array}{c}\cdot N \cdot \\
(\cdot / F \Delta-\cdot / A V)\end{array}$ & $\begin{array}{c}\cdot M " \\
(\cdot / \Delta \Delta-\cdot / N \ominus)\end{array}$ & $\Delta / \cdot \Lambda$ & $\begin{array}{c}\cdot N 1 \\
(\cdot / \Delta \&-\cdot / N s)\end{array}$ & $\begin{array}{c}. / \pi \wedge \\
(\cdot / \cdot \cdot 1)\end{array}$ & جهت داخلى آرمون SEBT (سائتىمت) \\
\hline $\begin{array}{c}. / 90 \\
(. / 4+\cdots / / 4)\end{array}$ & $\begin{array}{c}\cdot M " \\
(\cdot / \Delta \Delta-\cdot / / \& \xi)\end{array}$ & P/TO & $\begin{array}{c}. N F \\
(\cdot / \Delta q-\cdot / M)\end{array}$ & $\begin{array}{c}\cdot / A T \\
(<\cdot / \cdot+1)\end{array}$ & جهت خلفى داخلى آزمون SEBT (سانتىمتر) \\
\hline
\end{tabular}

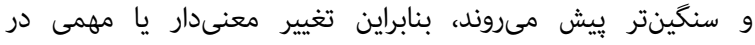

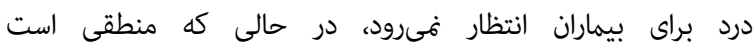

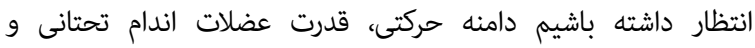

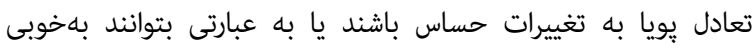

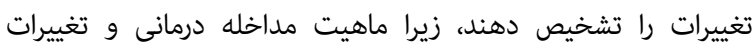

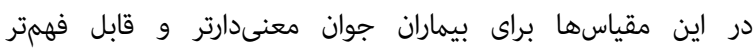

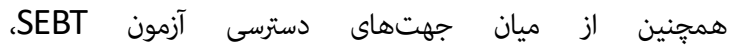

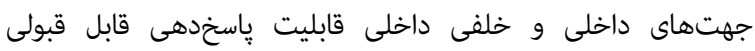

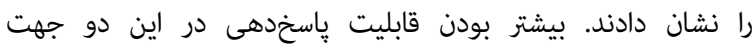

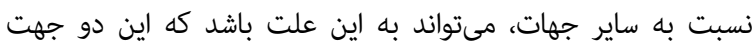

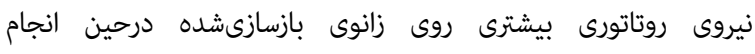

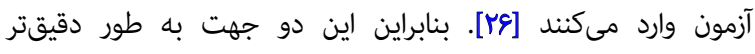

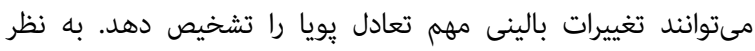

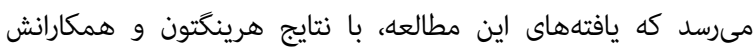

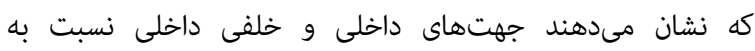
ساير جهات، قابليت بيشترى در افتراق مابين افراد مبتلا به آسيب داني

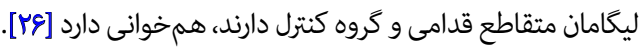

همجنين نقطه برش مطلوب كه از طريق منحنى ROC به دست
از اين دو بيامد مهم جهت مانيتورينگ بهبودى واقعى بيماران جوان و فعال در فضاى بالين و تحقيق بهره برد.

همجنين نتايج آناليز ROC و همبستكى نشان داد كه درد

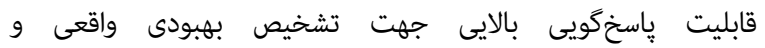

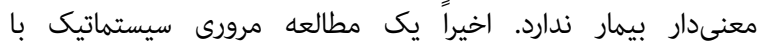

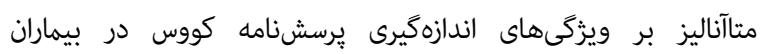

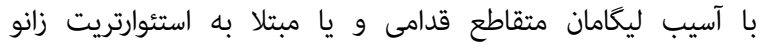

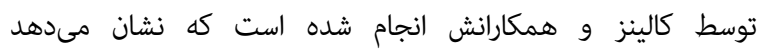

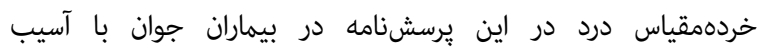

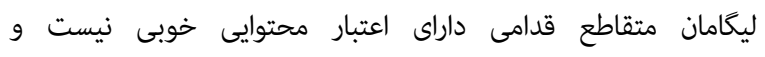

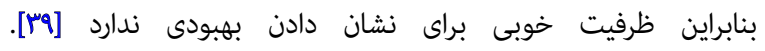

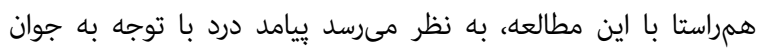

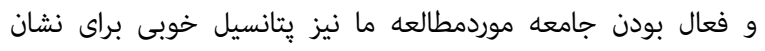

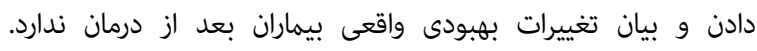

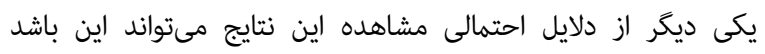

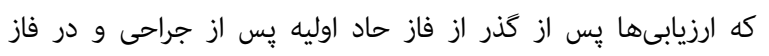

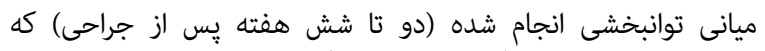

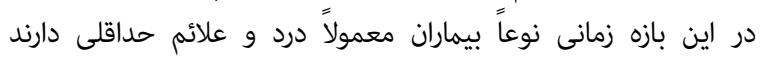

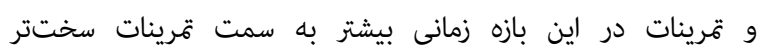


يريدن و غيره) در بيماران با بازسازى ليگامان متقاطع قدامى در فاز بازگشت به ورزش يرداخته شود.

ملاحظات اخلاقي

يميروي از اصول اخلاق يزووهش

اصول اخلاقى قاماً در اين مقاله رعايت شده است. شركت

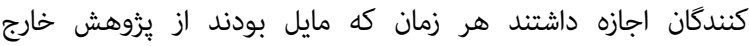

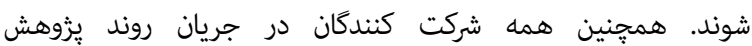

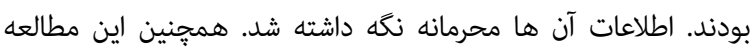

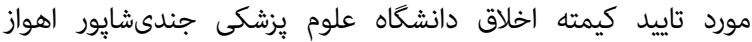
قرار كرفته است (كد:

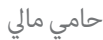
اين مطالعه بركرفته از رسالهى دكترى نويسنده اول در مركز

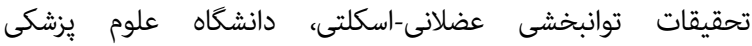

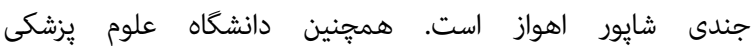
جندى شايور اهواز از مطالعه حمايت مالى كرده است.

\section{مشاركتنويسندكان}

مفهــوم ســازى: محمــد جعفــر شــاطرزاده يـزدى، حسـين

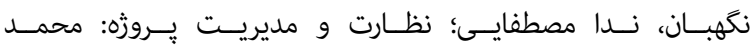

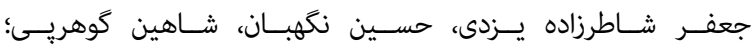

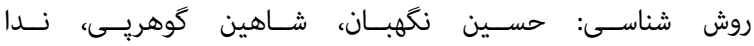

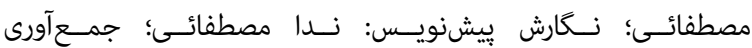

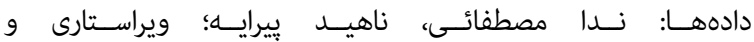

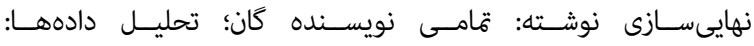

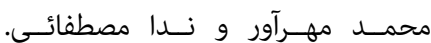

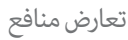
بنابر اظهار نويسندكان اين مقاله تعارض منافع ندارد.
مىآيد و به عنوان حداقل تغيير بالينى مهم در وضعيت سلامتى

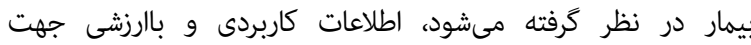

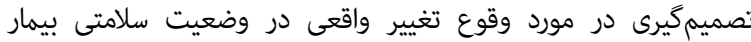

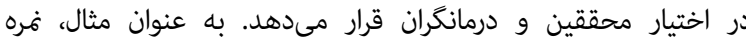

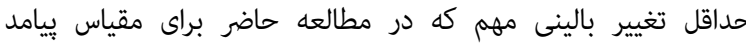

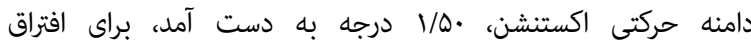

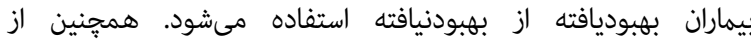

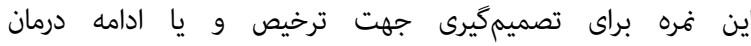

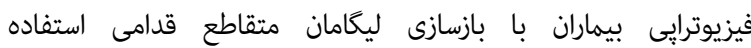

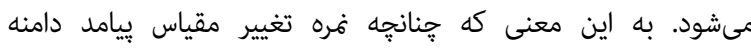

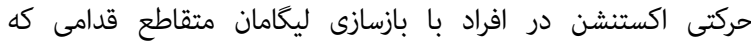

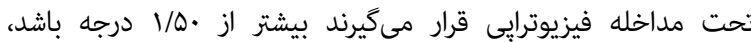

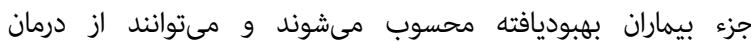

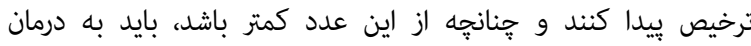

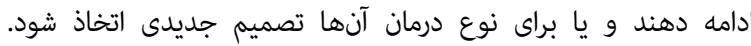
استفاده از اين كره در مطالعات و تحقيقات نيز كاربرد زيادى دارد.

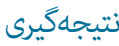

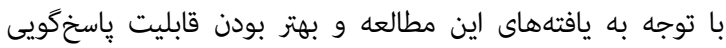

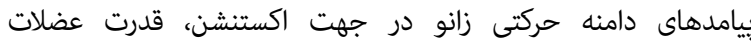

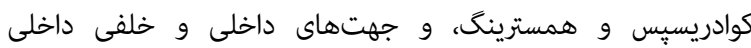

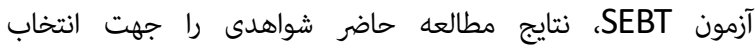

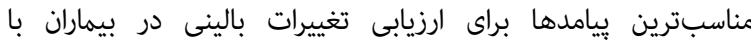

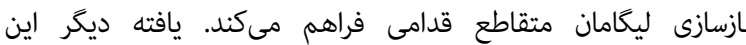

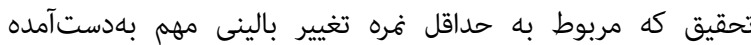

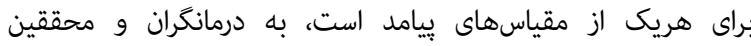

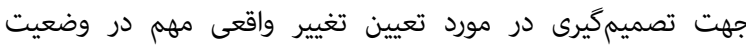

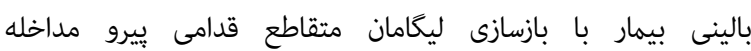
فيزيوترايى كمك خواهد كرد.

از آنجايى كه ليكرت هفتآيتمى مورداستفاده در مطالعه

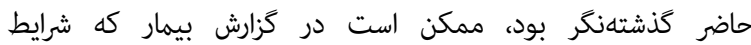
بعد و قبل از درمان را مقايسه مىكند، منجر به بروز خطا شوداد.

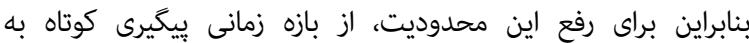

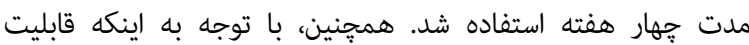

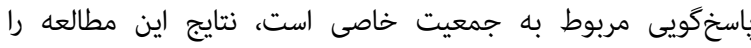

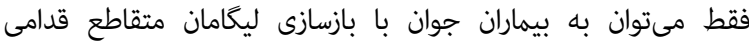

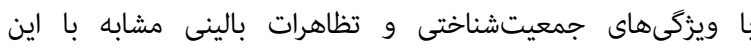

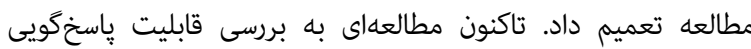

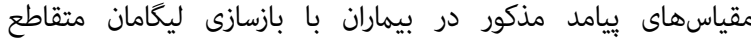

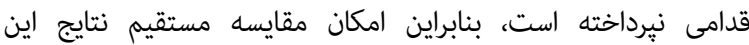
مطالعه با مطالعات ديكر وجود ندارد. ييشنهاد مىشود كه در مطالعات بعدى به بررسى قابليت

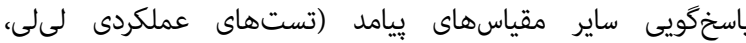




\section{References}

[1] Bonfim TR, Jansen Paccola CA, Barela JA. Proprioceptive and behavior impairments in individuals with anterior cruciate ligament reconstructed knees. Archives of Physical Medicine and Rehabilitation. 2003; 84(8):1217-23. [DOI:10.1016/S00039993(03)00147-3]

[2] Howells BE, Ardern CL, Webster KE. Is postural control restored following anterior cruciate ligament reconstruction? A systematic review. Knee Surgery, Sports Traumatology, Arthroscopy. 2011; 19(7):1168-77. [DOI:10.1007/s00167-011-1444-x]

[3] Logerstedt D, Lynch A, Axe MJ, Snyder-Mackler L. Symmetry restoration and functional recovery before and after anterior cruciate ligament reconstruction. Knee Surgery, Sports Traumatology, Arthroscopy. 2013; 21(4):859-68. [DOI:10.1007/s00167012-1929-2]

[4] Logerstedt D, Lynch A, Axe MJ, Snyder-Mackler L. Pre-operative quadriceps strength predicts IKDC2000 scores 6 months after anterior cruciate ligament reconstruction. The Knee. 2013; 20(3):208-12. [DOI:10.1016/j.knee.2012.07.011]

[5] Zouita Ben Moussa A, Zouita S, Dziri C, Ben Salah FZ. [Singleleg assessment of postural stability and knee functional outcome two years after anterior cruciate ligament reconstruction (EnglishFrench)]. Annals of Physical and Rehabilitation Medicine. 2009; 52(6):475-84. [DOI:10.1016/j.rehab.2009.02.006]

[6] Arna Risberg M, Lewek M, Snyder-Mackler L. A systematic review of evidence for anterior cruciate ligament rehabilitation: How much and what type? Physical Therapy in Sport. 2004; 5(3):125-45. [DOI:10.1016/j.ptsp.2004.02.003]

[7] Lobb R, Tumilty S, Claydon LS. A review of systematic reviews on anterior cruciate ligament reconstruction rehabilitation. Physical Therapy in Sport. 2012; 13(4):270-8. [DOI:10.1016/j. ptsp.2012.05.001]

[8] van Grinsven S, van Cingel REH, Holla CJM, van Loon CJM. Evidence-based rehabilitation following anterior cruciate ligament reconstruction. Knee Surgery, Sports Traumatology, Arthroscopy. 2010; 18(8):1128-44. [DOI:10.1007/s00167-009-1027-2]

[9] Cleland JA, Whitman JM, Houser JL, Wainner RS, Childs JD. Psychometric properties of selected tests in patients with lumbar spinal stenosis. The Spine Journal. 2012; 12(10):921-31. [DOI:10.1016/j.spinee.2012.05.004]

[10] Terwee CB, Bot SDM, de Boer MR, van der Windt DAWM, Knol DL, Dekker J, et al. Quality criteria were proposed for measurement properties of health status questionnaires. Journal of Clinical Epidemiology. 2007; 60(1):34-42. [DOI:10.1016/j. jclinepi.2006.03.012]

[11] Lehman LA, Velozo CA. Ability to detect change in patient function: Responsiveness designs and methods of calculation. Journal of Hand Therapy. 2010; 23(4):361-70. [DOI:10.1016/j. jht.2010.05.003]

[12] Pengel LHM, Refshauge KM, Maher CG. Responsiveness of pain, disability, and physical impairment outcomes in patients with low back pain. Spine. 2004; 29(8):879-83. [DOI:10.1097/00007632-200404150-00011]
[13] Sakulsriprasert P, Vachalathiti R, Vongsirinavarat M, Pichaisak W. Responsiveness of pain, active range of motion, and disability in patients with acute nonspecific low back pain. Hong Kong Physiotherapy Journal. 2011; 29(1):20-4. [DOI:10.1016/j.hkpj.2011.02.003]

[14] French HP, Fitzpatrick M, FitzGerald O. Responsiveness of physical function outcomes following physiotherapy intervention for osteoarthritis of the knee: An outcome comparison study. Physiotherapy. 2011; 97(4):302-8. [DOI:10.1016/j.physio.2010.03.002]

[15] Husted JA, Cook RJ, Farewell VT, Gladman DD. Methods for assessing responsiveness: A critical review and recommendations. Journal of Clinical Epidemiology. 2000; 53(5):459-68. [DOI:10.1016/ S0895-4356(99)00206-1]

[16] Kamper SJ, Maher CG, Mackay G. Global rating of change scales: A review of strengths and weaknesses and considerations for design. Journal of Manual \& Manipulative Therapy. 2009; 17(3):16370. [DOI:10.1179/jmt.2009.17.3.163]

[17] Briggs KK, Kocher MS, Rodkey WG, Steadman JR. Reliability, validity, and responsiveness of the Lysholm knee score and Tegner activity scale for patients with meniscal injury of the knee. The Journal of Bone \& Joint Surgery. 2006; 88(4):698-705. [DOI:10.2106/00004623-200604000-00003]

[18] Briggs KK, Lysholm J, Tegner Y, Rodkey WG, Kocher MS, Steadman JR. The reliability, validity, and responsiveness of the Lysholm score and Tegner activity scale for anterior cruciate ligament injuries of the knee: 25 years later. The American Journal of Sports Medicine. 2009; 37(5):890-7. [DOI:10.1177/0363546508330143]

[19] Roos EM, Roos HP, Ekdahl C, Lohmander LS. Knee injury and Osteoarthritis Outcome Score (KOOS)--validation of a Swedish version. Scandinavian Journal of Medicine \& Science in Sports. 1998; 8(6):439-48. [DOI:10.1111/j.1600-0838.1998.tb00465.x]

[20] Shaw T, Chipchase LS, Williams MT. A users guide to outcome measurement following ACL reconstruction. Physical Therapy in Sport. 2004; 5(2):57-67. [DOI:10.1016/S1466-853X(04)00019-7]

[21] Warren KJC, Chua CDM, Tagala AA, Cadiz BO, Maglanque NP. Validity and reliability of selected outcome measures used in rehabilitation for anterior cruciate ligament reconstruction: A literature review. Philippine Journal of Allied Health Sciences . 2006; 1:41-50. https://scholar.google.com/scholar?hl=en\&as_sdt=0\%2C 8 \&

[22] Gogia PP, Braatz JH, Rose SJ, Norton BJ. Reliability and validity of goniometric measurements at the knee. Physical Therapy. 1987; 67(2):192-5. [DOI:10.1093/ptj/67.2.192]

[23] Janssen JC, Le-Ngoc L. Intratester reliability and validity of concentric measurements using a new hand-held dynamometer. Archives of Physical Medicine and Rehabilitation. 2009; 90(9):1541-7. [DOI:10.1016/j.apmr.2009.02.021]

[24] Chamorro C, Armijo-Olivo S, De la Fuente C, Fuentes J, Javier Chirosa L. Absolute reliability and concurrent validity of hand held dynamometry and isokinetic dynamometry in the hip, knee and ankle joint: Systematic review and meta-analysis. Open medicine. 2017; 12(1):359-75. [DOI:10.1515/med-2017-0052]

[25] Le-Ngoc L, Janssen J. Validity and reliability of a hand-held dynamometer for dynamic muscle strength assessment. In: Kim CT, editor. Rehabilitation Medicine. London: IntechOpen; 2012. pp. 53-66. [DOI:10.5772/37688] 
[26] Herrington L, Hatcher J, Hatcher A, McNicholas M. A comparison of Star Excursion Balance Test reach distances between ACL deficient patients and asymptomatic controls. The Knee. 2009; 16(2):14952. [DOI:10.1016/j.knee.2008.10.004]

[27] Munro AG, Herrington LC. Between-session reliability of the Star Excursion Balance Test. Physical Therapy in Sport. 2010; 11(4):12832. [DOI:10.1016/j.ptsp.2010.07.002]

[28] Gribble PA, Hertel J, Plisky Ph. Using the Star Excursion Balance Test to assess dynamic postural-control deficits and outcomes in lower extremity injury: A literature and systematic review. Journal of Athletic Training. 2012; 47(3):339-57. [DOI:10.4085/1062-6050-47.3.08]

[29] Powden CJ, Dodds TK, Gabriel EH. The reliability of the Star Excursion Balance Test and lower quarter y-balance test in healthy adults: A systematic review. International Journal of Sports Physical Therapy. 2019; 14(5):683-94. [PMID] [PMCID]

[30] Stratford PW, Binkley JM, Riddle DL. Health status measures: Strategies and analytic methods for assessing change scores. Physical Therapy. 1996; 76(10):1109-23. [DOI:10.1093/ptj/76.10.1109]

[31] Heijne A, Werner S. Early versus late start of open kinetic chain quadriceps exercises after ACL reconstruction with patellar tendon or hamstring grafts: A prospective randomized outcome study. Knee Surgery, Sports Traumatology, Arthroscopy. 2007; 15(4):472-3. [DOI:10.1007/s00167-007-0313-0]

[32] Deyo RA, Centor RM. Assessing the responsiveness of functional scales to clinical change: An analogy to diagnostic test performance. Journal of Chronic Diseases. 1986; 39(11):897-906. [DOI:10.1016/0021-9681(86)90038-X]

[33] Houweling TAW. Reporting improvement from patient-reported outcome measures: A review. Clinical Chiropractic. 2010;13(1):15-22. [DOI:10.1016/j.clch.2009.12.003]

[34] Florkowski CM. Sensitivity, specificity, Receiver-Operating Characteristic (ROC) curves and likelihood ratios: Communicating the performance of diagnostic tests. The Clinical Biochemist Reviews. 2008; 29 Suppl 1(Suppl 1):S83-7. [PMID] [PMCID]

[35] Lehman LA, Sindhu BS, Shechtman O, Romero S, Velozo CA. A comparison of the ability of two upper extremity assessments to measure change in function. Journal of Hand Therapy. 2010; 23(1):31-40. [DOI:10.1016/j.jht.2009.09.006]

[36] Yazdi H, Moradi A, Sanaie A, Ghadi A. Does the hyperextension maneuver prevent knee extension loss after arthroscopic anterior cruciate ligament reconstruction? Journal of Orthopaedics and Traumatology. 2016; 17(4):327-31. [DOI:10.1007/s10195-016-0408-9]

[37] Keays SL, Bullock-Saxton J, Keays AC, Newcombe P. Muscle strength and function before and after anterior cruciate ligament reconstruction using semitendonosus and gracilis. The Knee. 2001; 8(3):229-34. [DOI:10.1016/S0968-0160(01)00099-0]

[38] de Jong SN, van Caspel DR, van Haeff MJ, Saris DBF. Functional assessment and muscle strength before and after reconstruction of chronic anterior cruciate ligament lesions. Arthroscopy. 2007; 23(1):21.E1-11. [DOI:10.1016/j.arthro.2006.08.024]

[39] Collins NJ, Prinsen CAC, Christensen R, Bartels EM, Terwee CB, Roos EM. Knee injury and Osteoarthritis Outcome Score (KOOS): Systematic review and meta-analysis of measurement properties. Osteoarthritis and Cartilage. 2016; 24(8):1317-29. [DOI:10.1016/j.joca.2016.03.010] 
This Page Intentionally Left Blank 